\title{
Parity violation in quasielastic electron scattering from closed-shell nuclei*
}

\author{
J.E. Amaro ${ }^{1,3}$, J.A. Caballero ${ }^{2}$, T.W. Donnelly ${ }^{1}$, A.M. Lallena ${ }^{3}$, E. Moya de Guerra ${ }^{2}$ \\ and J.M. Udías ${ }^{4}$ \\ ${ }^{1}$ Center for Theoretical Physics, Laboratory for Nuclear Science, and Dept. of Physics, \\ Massachusetts Institute of Technology, Cambridge, MA 02139, USA \\ ${ }^{2}$ Instituto de Estructura de la Materia, Consejo Superior de Investigaciones Científicas, \\ Serrano 123, Madrid 28006, Spain \\ ${ }^{3}$ Departamento de Física Moderna, Universidad de Granada, \\ E-18071 Granada, Spain \\ ${ }^{4}$ National Laboratory for Nuclear and High-Energy Physics, Section K (NIKHEF-K), \\ P.O. Box 41882, NL-1009 DB Amsterdam, The Netherlands \\ MIT-CTP-2472. nucl-th/9510006 October 1995. \\ Submitted to Nuclear Physics A
}

\begin{abstract}
The electromagnetic and weak neutral current matrix elements that enter in the analysis of parity-violating quasielastic electron scattering are calculated using a continuum nuclear shell model. New approximations to the on-shell relativistic one-body currents and relativistic kinematics for use in such models are developed and discussed in detail. Results are presented for three closed-shell nuclei of interest: ${ }^{16} \mathrm{O},{ }^{40} \mathrm{Ca}$ and ${ }^{208} \mathrm{~Pb}$. The current work concludes with a study of the sensitivity of the resulting parity-violating asymmetries to properties of the nucleon form factors including the possible strangeness content of the nucleon.
\end{abstract}

* This work is supported in part by funds provided by the U.S. Department of Energy (D.O.E.) under cooperative agreement \#DE-FC01-94ER40818, in part by DGICYT (Spain) under Contract Nos. PB92/0021-C02-01 and PB92-0927 and the Junta de Andalucía (Spain), in part by the European Commission under Contract ERBCHBICT920185, and in part by NATO Collaborative Research Grant \#940183. 


\section{Introduction}

Following the discovery of parity violation (PV) in nuclear beta decay, nuclei have played a central role in the investigation not only of the properties of the weak interaction but also of hidden properties of nucleon and nuclear structure. Prior to the discovery of weak neutral currents, investigations of parity violation in electromagnetic nuclear processes were focused on the study of asymmetries and polarizations in photonuclear absorption and decays [1]. The latter were understood as being caused by the parity impurities in nuclear states induced by the charge-changing parity-violating part of the nucleon-nucleon interaction mediated by meson exchanges [2]. This parity-mixing of nuclear states continues to be of interest and, for example, is at present one of the motivations of proposed experiments to search for nuclear anapole moments [3].

Soon after measurements of neutrino scattering at CERN [4 implied the existence of weak neutral currents, the vector/axial-vector character of these currents was confirmed by the observation of parity violation in electron-deuteron scattering at SLAC [5]. These experiments initiated a new era for the subject of parity violation in nuclei, and since then several experiments have been undertaken or proposed to measure PV asymmetries in electron-nucleus scattering (see Ref. [6] for discussion of the present situation). Two important experiments have been carried out so far on complex nuclei, elastic scattering from ${ }^{12} \mathrm{C}$ at Bates [7] and quasielastic (QE) scattering from ${ }^{9}$ Be at Mainz [8], yielding PV asymmetries that are, within experimental uncertainties, consistent with the Standard Model. Yet there is ample room open for speculation, and one of the main interests at present for PV electron-nucleus experiments lies in the possibility of extracting information on elusive parts of the nucleon form factors. In particular, as discussed at length in Ref. [6], there is at present considerable interest in studying various aspects of the nucleon's strangeness content, that is, its strangeness distributions or form factors.

Exploiting the rich variety of nuclei allows one to explore various features of the electroweak nucleon current and form factors complementary to those accesible via lepton-proton scattering. For instance, the above-mentioned experiment on ${ }^{12} \mathrm{C}$ performed at Bates [7] was designed to filter out the isoscalar electroweak coupling with hadronic matter. Or, as in the experiment on ${ }^{9}$ Be performed at Mainz [8], measuring parity violation in QE electron scattering has the advantage of involving large cross sections, in addition to that of involving various combinations of isoscalar, isovector, electric, and magnetic vector (and axial-vector) form factors, which can in principle be selected by appropiate choices of nuclei and of kinematics. The price one has to pay for this is that the nuclear many-body problem enters the picture, and ambiguities in the description of nuclear structure may render ambiguous the extraction of the various singlenucleon form factors. Obviously, an unambiguous extraction of nucleon form factors at least requires careful evaluation of the underlying mean-field-based description of the nuclear structure 
involved.

In Ref. [9] a study was carried out of the sensitivity to nucleon form factors (particularly to axial-vector and strangeness form factors) of PV response functions and asymmetries in QE electron scattering from ${ }^{12} \mathrm{C}$. The study was carried out within the context of the relativistic Fermi gas model (RFG) and then later extended in Refs. [10, 11, 12, 13 to more sophisticated models that incorporate specific classes of correlations and meson exchange current (MEC) effects. In other work reported in Ref. [14] an approach arising from models based on relativistic mean field theory has been pursued. As discussed in Ref. [13], for appropriately chosen observables it appears to be possible to extract useful information from measurements of the QE PV asymmetry in nuclei that bears on issues of the strangeness content of the the nucleon and its axial-vector, isovector current. Importantly, in the analyses to date the "confusion" from nuclear physics uncertainties appears not to obscure such extractions, despite the fact that reservations might be raised concerning the nuclear models used [15].

Indeed, the impulse approximation has been shown to be a good approximation for QE kinematics and final-state interactions are not expected to affect the PV asymmetries for high momentum transfers, so that the models used previously are quite likely to be capable of yielding the critical observables for extracting information on the single-nucleon form factors from the integrated PV asymmetry. However, it is still important to explore alternative approaches to the nuclear dynamics. Only if the appropriate observables are stable with respect to making different choices for the nuclear models is it then reasonable to expect that the single-nucleon content can be extracted; if different nuclear models yield significantly different results, then one must doubt the entire procedure at present, since each model has its special merit and no one approach includes all aspects of the problem (relativistic effects, many-body currents, correlations of all types - Hartree-Fock, RPA, et cetera).

Accordingly, in this work we extend the previous work by exploring the various PV observables within the context of a continuum shell model (CSM) description for comparison with the other approaches mentioned above. In particular, in this work the CSM is applied to three closed-shell nuclei, ${ }^{16} \mathrm{O},{ }^{40} \mathrm{Ca}$ and ${ }^{208} \mathrm{~Pb}$ where the approximate expressions used for the currents are known to hold best (see Sect. 2). This model has been thoroughly tested by Amaro et al. [16, 17, 18 for the computation of (parity-conserving) longitudinal and transverse responses in the region of the QE peak. In Ref. [16] a comparison was presented with results obtained using the continuum random phase approximation (RPA) model of Ref. [19, which has as a residual interaction the effective finite-range interaction derived from the nuclear matter polarization potential of Ref. [20]. It was found [16] that the CSM approach produces similar results at the $q$-values of concern here (the results are practically undistinguishable at $q=550 \mathrm{MeV} / \mathrm{c}$ ). In this past work the effects of meson-exchange currents were also investigated and it was seen in Ref. [17] that the net effect of MEC is very small except in the very high energy tail of the QE 
response where contributions of the two-particle emission channel dominate the $R^{T}$ response (as an example, for $q=550 \mathrm{MeV} / \mathrm{c}$ this starts to be important only at $\omega \sim 350 \mathrm{MeV}$ ). Thus the CSM is expected to be reliable as well for the computation of PV response functions in the vicinity of the QE peak. The issue of relevance for the present work is how different the various PV observables are in the CSM compared with those that result when other models such as the ones mentioned above are employed. Note that previous CSM modeling has been undertaken only at the non-relativistic level and only for the case of the EM responses [17, 18]; here we include relativity in the calculation and consider also the PV responses and asymmetries.

This paper is organized as follows. In Sect. 2 we very briefly review the formalism for PV electron-nucleus scattering reactions based on the material presented in a recent review article on the subject [6]. The discussion centers around several issues: (1) We review the essential expressions for the general nuclear response functions and PV asymmetry; (2) We consider the electroweak hadronic currents for on-shell nucleons and drawing on exact answers and various approximations to these quantities (whose details are presented in Appendix A) we develop the single-nucleon currents and their one-body consequences; (3) We summarize the continuum shell model that forms the basis of the present work (again, with some specifics relegated to Appendix B and other connections to the RFG model placed in Appendix C). In Sect. 3 we present the results obtained for the response functions and asymmetries for the cases of the three closed-shell nuclei ${ }^{16} \mathrm{O},{ }^{40} \mathrm{Ca}$ and ${ }^{208} \mathrm{~Pb}$. The discussions in that section open with a reexamination of the approximations made in the present work for relativistic aspects of currents and kinematics. In particular, we apply these to the Fermi gas model, where we know the exact answer (i.e., the RFG), as motivation for taking the same approach in the CSM. Following this the PV asymmetry is explored with the aim of quantifying the nuclear model uncertainties

inherent in attempts to extract information about the nucleon's strangeness and/or axial-vector content from PV QE electron scattering. Finally in Sect. 4 we summarize our conclusions.

\section{Summary of theory}

\subsection{Asymmetry and Nuclear Responses}

The general formalism for parity-violating electron scattering has been presented in detail in previous work (see Refs. [6, 21] and references therein). Therefore, in this subsection we summarize only those aspects of the reaction that are needed for the discussions that follow. As in Ref. [6], we limit our attention to the plane-wave Born approximation (PWBA) with single photon or $Z^{0}$ exchange. The processes we consider are represented in Fig. 1. Here, an electron with four-momentum $K^{\mu}=(\epsilon, \boldsymbol{k})$ and helicity $h$ is scattered through an angle $\theta_{e}$ to four-momentum $K^{\prime \mu}=\left(\epsilon^{\prime}, \boldsymbol{k}^{\prime}\right)$, exchanging a photon or a $Z^{0}$, where the transferred four-momentum in the pro- 
cess is given by $Q^{\mu}=\left(K-K^{\prime}\right)^{\mu}=(\omega, \boldsymbol{q})$. We follow the conventions of Ref. [22], including the invariant phase space factors that are neglected in some other work.

The basic quantity of interest here is the helicity asymmetry, defined as the ratio involving the difference and the sum of the electron scattering cross sections for positive and negative incoming electron helicities. The difference is parity-violating, while the sum is twice the usual parity-conserving electromagnetic (EM) cross section. When considering single-arm scattering of longitudinally polarized electrons from unpolarized nucleons and nuclei, the leading-order PV contribution arises from interferences between the two processes shown diagrammatically in Fig. 1. PV effects can also come from $Z^{0}$ exchange alone, but these are much smaller than the interference terms considered and thus are neglected here. Note that the $\gamma$-exchange amplitude (a) is purely vector, whereas the $Z^{0}$-exchange weak neutral current amplitude (b) has both vector $(\mathrm{V})$ and axial-vector $(\mathrm{A})$ contributions. The helicity-difference asymmetry may then be written

$$
\mathcal{A}=\frac{\frac{d \sigma^{+}}{d \Omega^{\prime} d \epsilon^{\prime}}-\frac{d \sigma^{-}}{d \Omega^{\prime} d \epsilon^{\prime}}}{\frac{d \sigma^{+}}{d \Omega^{\prime} d \epsilon^{\prime}}+\frac{d \sigma^{-}}{d \Omega^{\prime} d \epsilon^{\prime}}}=\mathcal{A}_{0} \frac{W_{\mathrm{pv}}}{W_{\mathrm{em}}}
$$

where the scale is set by the term

$$
\mathcal{A}_{0}=\frac{G\left|Q^{2}\right|}{2 \pi \alpha \sqrt{2}}
$$

with $G$ the Fermi coupling and $\alpha$ the fine structure constant.

The nuclear physics (hadronic) content in the problem is contained in the ratio of the parityviolating responses $\left(W_{\mathrm{pv}}\right)$ to the familiar parity-conserving EM responses $\left(W_{\mathrm{em}}\right)$. Within the PWBA, these are given by

$$
\begin{aligned}
& W_{\mathrm{em}}=v_{L} R^{L}+v_{T} R^{T} \\
& W_{\mathrm{pv}}=v_{L} R_{A V}^{L}+v_{T} R_{A V}^{T}+v_{T^{\prime}} R_{V A}^{T^{\prime}},
\end{aligned}
$$

where $L$ and $T$ denote longitudinal and transverse projections with respect to $\boldsymbol{q}$, respectively. The whole dependence on the electron kinematics is contained in the usual kinematic factors, $v_{L}=Q^{4} / q^{4}, v_{T}=\tan ^{2}\left(\theta_{e} / 2\right)-Q^{2} /\left(2 q^{2}\right)$ and $v_{T^{\prime}}=\tan \left(\theta_{e} / 2\right) \sqrt{\tan ^{2}\left(\theta_{e} / 2\right)-Q^{2} / q^{2}}$. The subscript $A V$ in the $\mathrm{PV}$ responses denotes interferences of axial-vector leptonic currents with vector hadronic currents and the reverse when written $V A$.

The five hadronic responses in Eqs. (3, (4) can be obtained from the components of the EM hadronic tensor $W_{\mathrm{em}}^{\mu \nu}$ in the case of the two pure EM responses $R^{L}$ and $R^{T}$, and from the hadronic tensor $W_{\mathrm{em} / \mathrm{nc}}^{\mu \nu}$ which involves interferences of EM and weak neutral currents (NC) for the three PV responses, $R_{A V}^{L}, R_{A V}^{T}$ and $R_{V A}^{T^{\prime}}$. By considering the laboratory coordinate system with the $z$-axis along $\boldsymbol{q}$, the $y$-axis along $\boldsymbol{k} \times \boldsymbol{k}^{\prime}$ and the $x$-axis in the electron scattering plane, the various 
hadronic response functions may be written as follows,

$$
\begin{aligned}
R^{L}=W_{\mathrm{em}}^{00} & =\sum_{f} \overline{\sum_{i}} \delta\left(E_{f}-E_{i}-\omega\right)\left|\left\langle f\left|\rho^{\mathrm{em}}\right| i\right\rangle\right|^{2} \\
R^{T}=W_{\mathrm{em}}^{x x}+W_{\mathrm{em}}^{y y} & =\sum_{f} \overline{\sum_{i} \delta} \delta\left(E_{f}-E_{i}-\omega\right)\left|\left\langle f\left|\boldsymbol{J}_{\perp}^{\mathrm{em}}\right| i\right\rangle\right|^{2},
\end{aligned}
$$

where the sum runs over all the final unobserved states and an average over initial states is performed. The terms $\rho^{\mathrm{em}}$ and $\boldsymbol{J}_{\perp}^{\mathrm{em}}$ represent the Fourier transforms of the EM nuclear charge and transverse current operators, respectively. Current conservation has been used to eliminate the $z$-component of the hadronic current.

For the PV responses we can write

$$
\begin{aligned}
R_{A V}^{L} & =-\frac{g_{A}}{2} W_{\mathrm{em} / \mathrm{nc}}^{00}=-\frac{g_{A}}{2} \sum_{f} \overline{\sum_{i}} \delta\left(E_{f}-E_{i}-\omega\right)\left\langle f\left|\rho^{\mathrm{em}}\right| i\right\rangle^{*}\left\langle f\left|\left(\rho^{\mathrm{nc}}\right)_{V}\right| i\right\rangle \\
R_{A V}^{T} & =-\frac{g_{A}}{2}\left(W_{\mathrm{em} / \mathrm{nc}}^{x x}+W_{\mathrm{em} / \mathrm{nc}}^{y y}\right) \\
& =-\frac{g_{A}}{2} \sum_{f} \overline{\sum_{i}} \delta\left(E_{f}-E_{i}-\omega\right)\left\langle f\left|\boldsymbol{J}_{\perp}^{\mathrm{em}}\right| i\right\rangle^{*} \cdot\left\langle f\left|\left(\boldsymbol{J}_{\perp}^{\mathrm{nc}}\right)_{V}\right| i\right\rangle \\
R_{V A}^{T^{\prime}} & =-\frac{i g_{V}}{2}\left(W_{\mathrm{em} / \mathrm{nc}}^{x y}-W_{\mathrm{em} / \mathrm{nc}}^{y x}\right) \\
& =-\frac{i g_{V}}{2} \sum_{f} \overline{\sum_{i}} \delta\left(E_{f}-E_{i}-\omega\right)\left[\left\langle f\left|\boldsymbol{J}^{\mathrm{em}}\right| i\right\rangle^{*} \times\left\langle f\left|\left(\boldsymbol{J}^{\mathrm{nc}}\right)_{A}\right| i\right\rangle\right] \cdot \frac{\boldsymbol{q}}{q} .
\end{aligned}
$$

Here the indices $V$ and $A$ refer to the vector and axial-vector contributions to the hadronic neutral current. The terms $\left(\rho^{\mathrm{nc}}\right)_{V}$ and $\left(\boldsymbol{J}_{\perp}^{\mathrm{nc}}\right)_{V}$ are the Fourier transforms of the NC vector nuclear charge and transverse current operators, respectively, while $\left(\boldsymbol{J}^{\mathrm{nc}}\right)_{A}$ is the Fourier transform of the axial-vector contribution in the $\mathrm{NC}$ operator.

The nature of the leptonic vertex appears here in the vector and axial-vector electron couplings, $g_{V}$ and $g_{A}$. The Standard model for the electroweak interaction at tree level is assumed in this work,

$$
\begin{aligned}
& g_{V}=-1+4 \sin ^{2} \theta_{W} \cong-0.092 \\
& g_{A}=1
\end{aligned}
$$

and $\theta_{W}$ the Weinberg angle $\left(\sin ^{2} \theta_{W} \cong 0.227\right.$, as discussed in Ref. [6]).

\subsection{Electroweak Hadronic Currents}

As noted from Eqs. (5)-(9), in order to calculate the EM and PV responses one needs to evaluate the pure EM and weak NC hadronic currents. Following the detailed study presented in Refs. [6, 21] and eliminating terms involving $(c, b, t)$ quarks, whose contributions to nuclear 
matrix elements of $\left(J_{\mu}^{\mathrm{nc}}\right)_{V / A}$ are supressed, the nuclear vector and axial-vector NC operators may be written as follows,

$$
\begin{aligned}
& \left(J_{\mu}^{\mathrm{nc}}\right)_{V}=\xi_{V}^{(T=1)} J_{\mu}^{\mathrm{em}}(T=1)+\sqrt{3} \xi_{V}^{(T=0)} J_{\mu}^{\mathrm{em}}(T=0)+\xi_{V}^{(0)} V_{\mu}^{(s)} \\
& \left(J_{\mu 5}^{\mathrm{nc}}\right)_{A}=\xi_{A}^{(T=1)} A_{\mu}^{(3)}+\xi_{A}^{(T=0)} A_{\mu}^{(8)}+\xi_{A}^{(0)} A_{\mu}^{(s)},
\end{aligned}
$$

where $J_{\mu}^{\mathrm{em}}(T=1) / J_{\mu}^{\mathrm{em}}(T=0)$ are the isovector/isoscalar EM currents, and $V_{\mu}^{(s)} \equiv \bar{s} \gamma_{\mu} s$ is the strange quark contribution in the vector $\mathrm{NC}$ current. The operators $A_{\mu}^{(a)}$ are given by $A_{\mu}^{(a)} \equiv \bar{q} \lambda^{a} \gamma_{\mu} \gamma_{5} q / 2$, where $q$ represents the triplet of quarks $(u, d, s)$, and the $\lambda^{a}, a=1 \ldots 8$ are the Gell-Mann SU(3) matrices. The strangeness content in the axial-vector NC current is given by $A_{\mu}^{(s)} \equiv \bar{s} \gamma_{\mu} \gamma_{5} s$. Finally, the $\xi_{V}$ 's and $\xi_{A}$ 's are couplings determined by the underlying electroweak gauge theory (see Refs. [6, 21]). We use the minimal Standard Model tree level couplings

$$
\begin{array}{lr}
\xi_{V}^{(0)}=-1 & \xi_{A}^{(0)}=1 \\
\sqrt{3} \xi_{V}^{(T=0)}=-4 \sin ^{2} \theta_{W} & \xi_{A}^{(T=0)}=0 \\
\xi_{V}^{(T=1)}=2-4 \sin ^{2} \theta_{W} & \xi_{A}^{(T=1)}=-2 .
\end{array}
$$

Single-nucleon matrix elements of the EM and weak NC currents shown previously are restricted by Lorentz covariance, together with parity and time reversal invariance to the following forms,

$$
\begin{aligned}
\left\langle N\left(P^{\prime}\right)\left|J_{\mu}^{\mathrm{em}}\right| N(P)\right\rangle & =\bar{u}\left(P^{\prime}\right)\left[F_{1} \gamma_{\mu}+i \frac{F_{2}}{2 M} \sigma_{\mu \nu} Q^{\nu}\right] u(P) \\
\left\langle N\left(P^{\prime}\right)\left|\left(J_{\mu}^{\mathrm{nc}}\right)_{V}\right| N(P)\right\rangle & =\bar{u}\left(P^{\prime}\right)\left[\tilde{F}_{1} \gamma_{\mu}+i \frac{\tilde{F}_{2}}{2 M} \sigma_{\mu \nu} Q^{\nu}\right] u(P) \\
\left\langle N\left(P^{\prime}\right)\left|\left(J_{\mu}^{\mathrm{nc}}\right)_{A}\right| N(p)\right\rangle & =\bar{u}\left(P^{\prime}\right)\left[\tilde{G}_{A} \gamma_{\mu}+\frac{\tilde{G}_{P}}{M} Q_{\mu}\right] \gamma_{5} u(P),
\end{aligned}
$$

where $u(P)$ and $u\left(P^{\prime}\right)$ are the single-nucleon wave functions properly normalized, $Q=P^{\prime}-P$ is the four momentum transfer to the nucleon and $M$ is the nucleon mass. From Eqs. (12, 13) and (15)-(17) one can write,

$$
\begin{aligned}
& \tilde{F}_{a}=\xi_{V}^{(T=1)} F_{a}^{(T=1)} \tau_{3}+\sqrt{3} \xi_{V}^{(T=0)} F_{a}^{(T=0)}+\xi_{V}^{(0)} F_{a}^{(s)}, \quad a=1,2 \\
& \tilde{G}_{a}=\xi_{A}^{(T=1)} G_{a}^{(3)} \tau_{3}+\xi_{A}^{(T=0)} G_{a}^{(8)}+\xi_{A}^{(0)} G_{a}^{(s)}, \quad a=A, P
\end{aligned}
$$

where $F_{a}^{(T=0,1)}$ denote the isoscalar and isovector EM Dirac and Pauli form factors of the nucleon, the $G_{a}^{(3,8)}$ are the triplet and octet axial-vector form factors, and $F_{a}^{(s)}$ and $G_{a}^{(s)}$ are the vector and axial-vector strange-quark form factors. In Eqs. (18) and (19) the terms involving $\tau_{3}$ are isovector while the rest are isoscalar. We will mainly use throughout this paper the Sachs form factors defined as: $G_{E}=F_{1}-\tau F_{2}$ and $G_{M}=F_{1}+F_{2}$, with $\tau \equiv\left|Q^{2}\right| / 4 M^{2}$. Analogously one can also define $\tilde{G}_{E}$ and $\tilde{G}_{M}$ from $\tilde{F}_{1}$ and $\tilde{F}_{2}$. Also, as discussed in Appendix A and Ref. [6], the pseudoscalar contributions are absent in PV electron scattering. 
In this work we follow the usual procedures of employing the on-shell single-nucleon currents to construct one-body EM and NC current operators. However, in contrast to some past work, we devise operators that retain important aspects of relativity. In Appendix A expressions for the exact on-shell operators for use between two-component spin spinors are summarized together with the approximations made in the present work when using these operators within the CSM. Below we repeat these approximate expressions for use in building the one-body nuclear current operators needed in treating PV electron scattering; specifically, only the vector time projections (EM and $\mathrm{NC}$ ) and transverse vector (EM and $\mathrm{NC}$ ) and axial-vector (NC) projections are required. As also discussed in Appendix A, since we consider only doublyclosed-shell nuclei, it is also a reasonable approximation to drop the spin-orbit contributions to the time projections, as we do in the following. With dimensionless variables $\kappa \equiv q / 2 M$ and $\tau$ defined above we then have

$$
\begin{aligned}
\rho^{\mathrm{em}} & =\frac{\kappa}{\sqrt{\tau}} G_{E} \\
\left(\rho^{\mathrm{nc}}\right)_{V} & =\frac{\kappa}{\sqrt{\tau}} \tilde{G}_{E} \\
\boldsymbol{J}^{\mathrm{em}} & =\frac{\sqrt{\tau}}{\kappa}\left(G_{E} \frac{\boldsymbol{p}+\boldsymbol{p}^{\prime}}{2 M}+i G_{M} \frac{\boldsymbol{\sigma} \times \boldsymbol{q}}{2 M}\right) \\
\left(\boldsymbol{J}^{\mathrm{nc}}\right)_{V} & =\frac{\sqrt{\tau}}{\kappa}\left(\tilde{G}_{E} \frac{\boldsymbol{p}+\boldsymbol{p}^{\prime}}{2 M}+i \tilde{G}_{M} \frac{\boldsymbol{\sigma} \times \boldsymbol{q}}{2 M}\right) \\
\left(\boldsymbol{J}^{\mathrm{nc}}\right)_{A} & =\sqrt{1+\tau} \tilde{G}_{A} \boldsymbol{\sigma},
\end{aligned}
$$

where for the three-vector components of the current only the transverse projections are to be employed - the longitudinal projections of the vector currents are given by the continuity equation and no longitudinal projections of the axial-vector current are required in descriptions of PV electron scattering. Therefore, within the IA, the hadronic (one-body) current operators are given by the following:

- Time Components

$$
\begin{aligned}
\rho^{\mathrm{em}} & =\frac{\kappa}{\sqrt{\tau}} \sum_{k=1}^{A} \mathrm{e}^{i \mathbf{q} \cdot \mathbf{r}_{k}}\left[G_{E}^{p} \frac{1+\tau_{3}^{k}}{2}+G_{E}^{n} \frac{1-\tau_{3}^{k}}{2}\right] \\
\left(\rho^{\mathrm{nc}}\right)_{V} & =\frac{\kappa}{\sqrt{\tau}} \sum_{k=1}^{A} \mathrm{e}^{i \mathbf{q} \cdot \mathbf{r}_{k}}\left[\tilde{G}_{E}^{p} \frac{1+\tau_{3}^{k}}{2}+\tilde{G}_{E}^{n} \frac{1-\tau_{3}^{k}}{2}\right]
\end{aligned}
$$

- Spatial Components (transverse projections only)

$$
\begin{aligned}
\boldsymbol{J}^{\mathrm{em}}= & -i \frac{\sqrt{\tau}}{\kappa} \sum_{k=1}^{A} \frac{\mathrm{e}^{i \mathbf{q} \cdot \mathbf{r}_{k}}}{2 M_{k}}\left[\left(G_{M}^{p} \frac{1+\tau_{3}^{k}}{2}+G_{M}^{n} \frac{1-\tau_{3}^{k}}{2}\right) \boldsymbol{q} \times \boldsymbol{\sigma}^{k}\right. \\
& \left.+\left(G_{E}^{p} \frac{1+\tau_{3}^{k}}{2}+G_{E}^{n} \frac{1-\tau_{3}^{k}}{2}\right)\left(2 \boldsymbol{\nabla}_{k}+i \boldsymbol{q}\right)\right]
\end{aligned}
$$




$$
\begin{aligned}
\left(\boldsymbol{J}^{\mathrm{nc}}\right)_{V}= & -i \frac{\sqrt{\tau}}{\kappa} \sum_{k=1}^{A} \frac{\mathrm{e}^{i \mathbf{q} \cdot \mathbf{r}_{k}}}{2 M_{k}}\left[\left(\tilde{G}_{M}^{p} \frac{1+\tau_{3}^{k}}{2}+\tilde{G}_{M}^{n} \frac{1-\tau_{3}^{k}}{2}\right) \boldsymbol{q} \times \boldsymbol{\sigma}^{k}\right. \\
& \left.+\left(\tilde{G}_{E}^{p} \frac{1+\tau_{3}^{k}}{2}+\tilde{G}_{E}^{n} \frac{1-\tau_{3}^{k}}{2}\right)\left(2 \boldsymbol{\nabla}_{k}+i \boldsymbol{q}\right)\right] \\
\left(\boldsymbol{J}^{\mathrm{nc}}\right)_{A}= & \sqrt{1+\tau} \sum_{k=1}^{A} \mathrm{e}^{i \mathbf{q} \cdot \mathbf{r}_{k}}\left[\tilde{G}_{A}^{p} \frac{1+\tau_{3}^{k}}{2}+\tilde{G}_{A}^{n} \frac{1-\tau_{3}^{k}}{2}\right] \boldsymbol{\sigma}^{k},
\end{aligned}
$$

where $\mathbf{r}_{k}$ is the position of the $k$-th nucleon, while $\boldsymbol{\sigma}^{k}$ and $\tau_{3}^{k}$ are the Pauli spin and (third component) isospin matrices for the $k$-th nucleon. As is standard we refer to the contributions proportional to $G_{M}$ and $\tilde{G}_{M}$ as magnetization contributions, and to those proportional to $G_{E}$ and $\tilde{G}_{E}$ as convection contributions.

\subsection{Nucleon Form Factors}

As seen in the previous section, the EM and NC current operators are given in terms of the familiar EM form factors, $G_{E}^{p, n}$ and $G_{M}^{p, n}$, and the weak NC ones, $\tilde{G}_{E}^{p, n}, \tilde{G}_{M}^{p, n}$ and $\tilde{G}_{A}^{p, n}$. Note, however, that by using Eqs. (18,19) one can express these weak NC form factors in terms of the purely EM ones $G_{E, M}^{p, n}$, the axial-vector cases, $G_{A}^{p, n}$, and the form factors $G_{E, M, A}^{(s)}$ that enter when the nucleon has nonzero $s \bar{s}$ strangeness components. At tree level we obtain for the nucleon weak form factors the following expressions (see Ref. [6]):

$$
\begin{aligned}
\tilde{G}_{E, M}^{p, n} & =\left(1-4 \sin ^{2} \theta_{W}\right) G_{E, M}^{p, n}-G_{E, M}^{n, p}-G_{E, M}^{(s)} \\
\tilde{G}_{A}^{p, n} & =\mp 2 G_{A}^{(3)}+G_{A}^{(s)} .
\end{aligned}
$$

From these results one easily sees that the strangeness form factors have an isoscalar character. We must also have $G_{E}^{(s)}=0$ for $Q^{2}=\omega^{2}-q^{2}=0$, since the nucleon has no net strangeness, whereas the $\tau=0$ values of the remaining strangeness form factors may be nonzero at $Q^{2}=0$. In the case of the axial-vector weak neutral current form factor $\tilde{G}_{A}$, all of the isoscalar dependence is contained in the strangeness form factor $G_{A}^{(s)}$, while the remaining term $G_{A}^{(3)}$ is purely isovector $(T=1)$. Note that this result is only valid at lowest order in the Standard Model where we have $\xi_{A}^{(T=0)}=0$. Therefore, in the absence of strange quarks, the isoscalar axial-vector form factor $\tilde{G}_{A}^{(T=0)}$ would vanish at tree-level (for a more detailed discussion of this subject see Refs. [6, 21]). There exist different conventions for the NC couplings; the inter-relationships between them are presented in Ref. [6].

In order to evaluate potential PV electron scattering experiments, it is mandatory to characterize the $Q^{2}$-dependence of the various nucleon form factors. In Sect. 3.3 we present a detailed study of the sensitivity shown by the asymmetries to specific aspects of the nucleon form factors. In particular, we compare the results obtained by calculating the EM form factors using the 
so-called Galster parameterization (see Refs. [6, 9, 23]):

$$
\begin{aligned}
G_{E}^{p} & =G_{V}^{D} \\
G_{M}^{p} & =\mu_{p} G_{V}^{D} \\
G_{E}^{n} & =-\mu_{n} \tau G_{V}^{D} \xi_{n} \\
G_{M}^{n} & =\mu_{n} G_{V}^{D},
\end{aligned}
$$

with $G_{V}^{D}(\tau)=\left[1+\lambda_{V}^{D} \tau\right]^{-2}, \xi_{n}(\tau)=\left[1+\lambda_{n} \tau\right]^{-1}, \lambda_{V}^{D}=4.97$ and $\lambda_{n}=5.6$. In Sect. 3.3 we consider variations of $\lambda_{n}$.

Following Refs. [6, 21] the isovector axial-vector form factor is given by

$$
G_{A}^{(T=1)}=-g_{A} G_{A}^{D}
$$

with $g_{A}=1.262$, and $G_{A}^{D}(\tau)=\left[1+\lambda_{A}^{D} \tau\right]^{-2}$ is the axial-vector dipole form factor. In this work we have assumed the value $\lambda_{A}^{D}=3.32$ corresponding to a dipole mass of $1 \mathrm{GeV}$. Effects due to a renormalization of $g_{A}$ can be quite significant in neutral current processes. In particular, in the case of electron scattering, uncertainties in theoretical estimates of higher-order contributions may be on the order of $\pm 10 \%$ (for a more complete discussion of these issues see Refs. [6, 21]). Again, in Sect. 3.3 we return to consider variations in the magnitude of $g_{A}$.

Finally, generalizing the Galster parameterization, the (purely isoscalar) strange form factors can be written as

$$
\begin{aligned}
G_{E}^{(s)} & =\rho_{s} \tau G_{V}^{D} \xi_{E}^{(s)} \\
G_{M}^{(s)} & =\mu_{s} G_{V}^{D} \xi_{M}^{(s)} \\
G_{A}^{(s)} & =g_{A}^{(s)} G_{A}^{D} \xi_{A}^{(s)}
\end{aligned}
$$

The values chosen for $\rho_{s}$ and $\mu_{s}$ will be discussed in Sect. 3.3, whereas the remaining form factor $G_{A}^{(s)}$ will be ignored in the present work as its effect in QE electron scattering is expected to be very small [6]. One should note that the choice given by Eqs. (37)-(39) is somewhat arbitrary, except for the $\tau \rightarrow 0$ behaviour of $G_{E}^{(s)}(\tau)$ dictated by the fact that the nucleon has no net strangeness. Given that no experimental information at all exists on $G_{E, M}^{(s)}$, while very little is known about $G_{A}^{(s)}$ (see Ref. [6]), Eqs. (37)-(39) simply show a straightforward extension of the pure EM form factor equations to characterize the present lack of knowledge in these form factors. Results of future measurements may require a different choice of parameterization. In this work, we describe the leading, non-trivial $Q^{2}$-dependence of $G_{M, E, A}^{(s)}$ via the parameters $\mu_{s}$, $\rho_{s}$ and $g_{A}^{(s)}$, respectively, and possible deviations of the high- $\tau$ dipole fall-off by the functions $\xi_{E, M, A}^{(s)}$ that show a $\tau$-dependence in the form $\xi_{E, M, A}^{(s)}(\tau)=\left(1+\lambda_{E, M, A}^{(s)} \tau\right)^{-1}$. The parameters $\lambda_{E, M, A}^{(s)}$ are, as yet, unconstrained. Our focus in the present work is entirely on the region where $\tau<0.3$. 


\subsection{Nuclear Shell model}

As has been already mentioned in previous sections, in this paper we restrict ourselves to the Impulse Approximation (IA), i.e., we only consider one-body current operators. The study of MEC will be presented in a forthcoming publication. In this context, apart from different prescriptions for the nucleon form factors, one must also model the nuclear structure in obtaining the nuclear matrix elements that enter in the various EM and PV response functions. In this subsection we briefly discuss the nuclear structure model used to describe the ground and excited states of the nuclei under consideration. In this work we restrict our attention to nuclei with doubly-closed shells, ${ }^{16} \mathrm{O},{ }^{40} \mathrm{Ca}$ and ${ }^{208} \mathrm{~Pb}$, taking the initial states $|i\rangle \equiv|0\rangle$ to be given simply by the degenerate ground-state wave functions of such nuclei, whereas the final states $|f\rangle$ are represented by $1 \mathrm{p}-1 \mathrm{~h}$ excitations (within the IA).

In the following we summarize the basic features of the CSM. The particle and hole, proton and neutron wave functions are solutions of the radial Schrödinger equation with a mean potential of Woods-Saxon type

$$
V(r)=\frac{-V_{0}}{1+\exp \left(r-R_{0}\right) / a_{0}}+\frac{1}{m_{\pi}^{2}} \frac{1}{r} \frac{d}{d r}\left(\frac{-V_{l s}}{1+\exp \left(r-R_{l s}\right) / a_{l s}}\right) \boldsymbol{l} \cdot \boldsymbol{\sigma}+V_{\text {Coul }},
$$

where $m_{\pi}$ is the pion mass, and $V_{\text {Coul }}$ is the Coulomb potential of a uniform charge distribution with radius $R_{\text {Coul }}$ and charge $(Z-1) e$. Obviously, the Coulomb potential only interacts in the case of protons. The parameters are fixed to have a good description of the empirical singleparticle energies near the Fermi level and of the experimental ground-state charge density. We take $R_{\text {Coul }}=R_{0}$. The rest of parameters are given in Table 1 and have been taken from Refs. [18, 24, 25]. In previous work [16, 17, 18] we have analyzed the sensitivity of the $R_{L}$ and $R_{T}$ responses to variations of these parameters.

The asymptotic form of the particle radial wave functions of momentum $k=\sqrt{2 M \epsilon}$ and angular momenta $l, j$ is

$$
R_{\epsilon l j}(r) \sim \sqrt{\frac{2 M}{\pi k}} \frac{1}{r} \sin \left(k r-\eta \log 2 k r-l \frac{\pi}{2}+\sigma_{l}+\delta_{l j}\right),
$$

where $\delta_{l j}$ is the nuclear phase shift, $\sigma_{l}$ is the Coulomb phase shift (which is absent for neutrons), and $\eta$ is the Coulomb parameter $M(Z-1) e^{2} / k$ for protons and zero for neutrons; the normalization of the wave functions is then

$$
\left\langle R_{\epsilon l j} \mid R_{\epsilon^{\prime} l j}\right\rangle=\delta\left(\epsilon-\epsilon^{\prime}\right)
$$

Special care is needed when the energy is small or very large, when the angular momentum $l$ is very large, and when the charge $Z$ is very large, since then the computation of the Coulomb wave functions is known to be somewhat delicate. 
In this approach, since we only consider inclusive electron scattering, the nuclear final state is taken to be an eigenstate of the total angular momentum of the many-particle system, i.e.,

$$
|f\rangle \equiv|\alpha, J\rangle=\left|p, h^{-1} ; J\right\rangle=\left[a_{p}^{\dagger} \otimes b_{h}^{\dagger}\right]_{J}|0\rangle,
$$

where $a_{p}^{\dagger}$ creates a particle in the continuum, namely state $|p\rangle=\left|\epsilon_{p} l_{p} j_{p}\right\rangle$ with $\epsilon_{p}>0$, while $b_{h}^{\dagger}$ creates a hole in the core $|0\rangle$ corresponding to destroying particle state $\left|n_{h} l_{h} j_{h}\right\rangle$. Then, for a fixed value of the excitation energy the nuclear excited state is calculated by summing over all of the particle-hole pairs $\left(\sum_{\alpha}\right)$ that are allowed by the angular momentum selection rules and energy conservation. The calculation of the responses requires the sum over all the excited states (see Refs. [16, 17, 18, 26] for details concerning the calculations).

When considering initial and final nuclear states with good angular momentum quantum numbers it is convenient to expand the hadronic currents in terms of multipole projections of the charge and transverse three-vector current operators:

$$
\begin{aligned}
\rho^{\mathrm{em}} & =\sqrt{4 \pi} \sum_{J \geq 0}(-i)^{J} \hat{J} M_{0}^{J} \\
J_{m}^{\mathrm{em}} & =-\sqrt{2 \pi} \sum_{J \geq 1}(-i)^{J} \hat{J}\left[T_{m}^{E J}+m T_{m}^{M J}\right],
\end{aligned}
$$

with $m= \pm 1$. We use the notation $\hat{J} \equiv \sqrt{2 J+1}$. Note that the longitudinal component of the three-current can be obtained from the charge current by using current conservation.

The above multipole decompositions can be applied to the EM currents, $\rho^{\mathrm{em}}, \boldsymbol{J}^{\mathrm{em}}$, as well as to the vector and axial-vector weak neutral currents, $\left(\rho^{\mathrm{nc}}\right)_{V},\left(\boldsymbol{J}^{\mathrm{nc}}\right)_{V},\left(\rho^{\mathrm{nc}}\right)_{A},\left(\boldsymbol{J}^{\mathrm{nc}}\right)_{A}$. The corresponding Coulomb and transverse electric and magnetic multipole operators are given as usual by

$$
\begin{aligned}
M_{m}^{J} & =\int d \boldsymbol{r} j_{J}(q r) Y_{J}^{m}(\hat{\boldsymbol{r}}) \rho(\boldsymbol{r}) \\
T_{m}^{E J} & =\frac{1}{q} \int d \boldsymbol{r}\left\{\boldsymbol{\nabla} \times\left[j_{J}(q r) \boldsymbol{Y}_{J J}^{m}(\hat{\boldsymbol{r}})\right]\right\} \cdot \boldsymbol{J}(\boldsymbol{r}) \\
T_{m}^{M J} & =\int d \boldsymbol{r} j_{J}(q r) \boldsymbol{Y}_{J J}^{m}(\hat{\boldsymbol{r}}) \cdot \boldsymbol{J}(\boldsymbol{r}),
\end{aligned}
$$

with $j_{J}(q r)$ the spherical Bessel functions, $Y_{J}^{m}(\hat{\boldsymbol{r}})$ the spherical harmonics and $\boldsymbol{Y}_{J J}^{m}(\hat{\boldsymbol{r}})$ the vector spherical harmonics. Here $\rho(\boldsymbol{r})$ and $\boldsymbol{J}(\boldsymbol{r})$ stand for any of the nuclear EM, vector NC and axialvector NC current operators.

Introducing the above multipole analysis in the general expressions for the response functions (Eqs. (5) -(9)) and making use of the Wigner-Eckart theorem, it is straightforward to obtain the following expressions:

$$
R^{L}(q, \omega)=4 \pi \sum_{J \geq 0} \sum_{\alpha} \delta\left(E_{\alpha}-E_{0}-\omega\right)\left|\left\langle\alpha, J\left\|M_{\mathrm{em}}^{J}\right\| 0\right\rangle\right|^{2}
$$




$$
\begin{aligned}
R^{T}(q, \omega) & =4 \pi \sum_{J \geq 1} \sum_{\alpha} \delta\left(E_{\alpha}-E_{0}-\omega\right)\left|\left\langle\alpha, J\left\|T_{\mathrm{em}}^{E J}+i T_{\mathrm{em}}^{M J}\right\| 0\right\rangle\right|^{2} \\
R_{A V}^{L}(q, \omega) & =-2 \pi g_{A} \sum_{J \geq 0} \sum_{\alpha} \delta\left(E_{\alpha}-E_{0}-\omega\right)\left\langle\alpha, J\left\|M_{\mathrm{em}}^{J}\right\| 0\right\rangle^{*}\left\langle\alpha, J\left\|\left(M_{\mathrm{nc}}^{J}\right)_{V}\right\| 0\right\rangle \\
R_{A V}^{T}(q, \omega) & =-2 \pi g_{A} \sum_{J \geq 1} \sum_{\alpha} \delta\left(E_{\alpha}-E_{0}-\omega\right)\left[\left\langle\alpha, J\left\|T_{\mathrm{em}}^{E J}\right\| 0\right\rangle^{*}\left\langle\alpha, J\left\|\left(T_{\mathrm{nc}}^{E J}\right)_{V}\right\| 0\right\rangle\right. \\
& \left.+\left\langle\alpha, J\left\|T_{\mathrm{em}}^{M J}\right\| 0\right\rangle^{*}\left\langle\alpha, J\left\|\left(T_{\mathrm{nc}}^{M J}\right)_{V}\right\| 0\right\rangle\right] \\
R_{V A}^{T^{\prime}}(q, \omega) & =-2 \pi g_{V} \sum_{J \geq 1} \sum_{\alpha} \delta\left(E_{\alpha}-E_{0}-\omega\right)\left[\left\langle\alpha, J\left\|T_{\mathrm{em}}^{E J}\right\| 0\right\rangle^{*}\left\langle\alpha, J\left\|\left(T_{\mathrm{nc}}^{M J}\right)_{A}\right\| 0\right\rangle\right. \\
& \left.+\left\langle\alpha, J\left\|T_{\mathrm{em}}^{M J}\right\| 0\right\rangle^{*}\left\langle\alpha, J\left\|\left(T_{\mathrm{nc}}^{E J}\right)_{A}\right\| 0\right\rangle\right],
\end{aligned}
$$

where we have used the notation, $M_{\mathrm{em}}^{J}, T_{\mathrm{em}}^{E J}$ and $T_{\mathrm{em}}^{M J}$ for the EM multipole operators, $\left(M_{\mathrm{nc}}^{J}\right)_{V}$, $\left(T_{\mathrm{nc}}^{E J}\right)_{V}$ and $\left(T_{\mathrm{nc}}^{M J}\right)_{V}$ for the multipole operators associated with the vector NC four-current, and $\left(T_{\mathrm{nc}}^{E J}\right)_{A}$ and $\left(T_{\mathrm{nc}}^{M J}\right)_{A}$ for the ones corresponding to the transverse axial-vector NC current.

Note that the Coulomb and electric operators have parity $(-1)^{J}$ in the case of EM and weak $\mathrm{NC}$ vector currents, but they have parity $(-1)^{J+1}$ for the axial-vector current. On the other hand, the magnetic operator has parity $(-1)^{J+1}$ for the EM and vector NC currents, and $(-1)^{J}$ for the axial-vector one.

Explicit expressions for the reduced matrix elements appearing in Eqs. (49 53) are given in Appendix B. It is worth pointing out that the following relationships hold for the approximate current matrix elements:

$$
\begin{aligned}
\left\langle\alpha, J\left\|\left(T_{\mathrm{nc}}^{E J}\right)_{A}\right\| 0\right\rangle & =\sqrt{1+\frac{1}{\tau}} \frac{\tilde{G}_{A}}{G_{M}}\left\langle\alpha, J\left\|T_{\mathrm{em}}^{M J}\right\| 0\right\rangle_{\mathrm{mag}} \\
& =\sqrt{1+\frac{1}{\tau} \frac{\tilde{G}_{A}}{\tilde{G}_{M}}\left\langle\alpha, J\left\|\left(T_{\mathrm{nc}}^{M J}\right)_{V}\right\| 0\right\rangle_{\mathrm{mag}}} \\
\left\langle\alpha, J\left\|\left(T_{\mathrm{nc}}^{M J}\right)_{A}\right\| 0\right\rangle & =\sqrt{1+\frac{1}{\tau}} \frac{\tilde{G}_{A}}{G_{M}}\left\langle\alpha, J\left\|T_{\mathrm{em}}^{E J}\right\| 0\right\rangle_{\mathrm{mag}} \\
& =\sqrt{1+\frac{1}{\tau}} \frac{\tilde{G}_{A}}{\tilde{G}_{M}}\left\langle\alpha, J\left\|\left(T_{\mathrm{nc}}^{E J}\right)_{V}\right\| 0\right\rangle_{\mathrm{mag}}
\end{aligned}
$$

where here "mag" denotes the magnetization current. Theoretically the sums in Eqs. (49)-(53) extend to $J_{\max }=\infty$, while in practice we add together only a finite number of multipoles. In general we use a value of $J_{\max }$ that is greater than that needed for convergence, $J_{\text {conv }}$, specifically the values given in Table 2 .

We must take into account the resonant part of the excitation spectrum at low energy. In addition we need to integrate the responses to compute the sum-rule asymmetry given in Eq. (57). Thus we need to compute the responses with a small step size, from emission threshold up to a sufficiently high energy $\omega_{\max }$ that the responses become negligible compared with their 
peak values. In particular for $q=300,500$ and $700 \mathrm{MeV} / \mathrm{c}$ we use as step sizes 1,2 and $5 \mathrm{MeV}$ and $\omega_{\max }=200,350$ and $500 \mathrm{MeV}$, respectively.

At low $\omega$ resonances in the Woods-Saxon potential produce rapid oscillations and sharp peaks in the responses, whose overall contribution we take into account by convoluting the responses with a Gaussian weight function $f(\omega) \propto \exp \left(-\omega^{2} / \Gamma^{2}\right)$ of width $\Gamma$ (in all cases we have used $\Gamma=5 \mathrm{MeV})$. So what we show in the plots is not actually the shell model response $R_{\mathrm{SM}}(q, \omega)$, but the "smoothed" response $R_{\Gamma}(q, \omega)$, given by

$$
R_{\Gamma}(q, \omega) \equiv \int_{-\infty}^{\infty} d \omega^{\prime} f\left(\omega-\omega^{\prime}\right) R_{\mathrm{SM}}\left(q, \omega^{\prime}\right)
$$

In the next section we compare the results obtained within the present model to those obtained with the RFG. To facilitate the discussion there, we summarize in Appendix $\mathrm{C}$ the expressions for the five response functions within the relativistic Fermi Gas Model together with the non-relativistic limits.

\section{Results}

In this section we present and study in detail the results obtained for the response functions and asymmetry defined in previous section and appendices of the present paper. An important goal in this work has been to explore the sensitivities that the various observables display to different nuclear models, as well as to properties of the single-nucleon form factors. Concerning the former, our motivation has been to investigate whether or not shell effects show up in the observables under consideration and to explore these nuclear model dependences using an approach that differs from that employed in past work in this area. In particular, as discussed above, we have argued that the CSM augmented by the incorporation of an approximate relativistic one-body current and relativistic kinematics should provide a reasonable model for inclusive mediumenergy QE electron scattering. Our aim in the present section is to present results that support this approach and then to proceed to investigate aspects of the single-nucleon structure physics as revealed in PV QE electron scattering.

To set a clear scale for the model dependences (and therefore the ambiguities) in the response functions and PV asymmetry, we have performed calculations for different values of the momentum transfer ranging from $q=300 \mathrm{MeV} / \mathrm{c}$ up to $1000 \mathrm{MeV} / \mathrm{c}$ using the CSM described in Sect. 2.4. In so-doing we deal with situations in which non-relativistic calculations are expected to be reliable, and others in which a relativistic analysis of the reaction mechanism is mandatory. Results for three closed-shell nuclei, ${ }^{16} \mathrm{O},{ }^{40} \mathrm{Ca}$ and ${ }^{208} \mathrm{~Pb}$, are presented. Naturally, the PWBA used in this work is not directly applicable in modeling the response functions for nuclei as heavy as lead. In such high- $Z$ cases the electron distortion can be taken into account by obtaining numerical solutions to the Dirac equation with a Coulomb potential. In the present 
paper our main focus is placed on the PV asymmetry which, being proportional to a ratio of helicity difference and sum cross sections, is less affected by distortion. Accordingly, for the model-to-model comparisons made here the PWBA should prove adequate.

Throughout this work, the nucleon's EM form factors are taken to be given by the simple parameterizations discussed in Sect. 2.3. Since it is expected that these functions will be studied intensively at most medium- to high-energy electron scattering facilities over the next few years, we have not pursued any further analysis of the sensitivity of the PV asymmetry to this aspect of the problem other than to present one figure showing the sensitivity to variations of $G_{E}^{n}$; this issue can easily be re-examined when new data become available from measurements of parityconserving polarization-dependent electron scattering from the proton, deuteron and ${ }^{3} \mathrm{He}$.

We begin by reproducing the observables in the RFG model studied in previous work 9 , 10, 11, 12, 13 (see Appendix C) to quantify further the approximations made in our present treatment of the CSM. Importantly, we show below that the current developed in Appendix A provides an excellent approximation to the exact on-shell results when used within the context of the Fermi gas model, confirming those past studies. The same approach is then used in the CSM to proceed to an investigation of the nuclear model dependences expected for PV observables.

\subsection{Quasielastic responses in the RFG model}

The RFG model has been summarized in Appendix $\mathrm{C}$ and several approximations to the singlenucleon current discussed in Appendix A. In the present subsection we present results using this model to help in assessing the quality of those approximations for use later in the CSM.

In Fig. 2 we show the EM response functions $R^{L}$ and $R^{T}$ for the case of ${ }^{40} \mathrm{Ca}$ at $q=500,700$ and $1000 \mathrm{MeV} / \mathrm{c}$. The solid curves result from using the fully relativistic model obtained with the exact on-shell current operators given in Eqs. (65 70), i.e., using the formalism presented in Ref. [10]. Those shown as long-dashed curves correspond to making the approximations for the current operators given in Eqs. (20) and (22). Moreover in this latter case we have begun with the non-relativistic Fermi gas model for the rest of the problem (i.e., other than the currents) and proceeded to make the $\lambda \equiv \omega / 2 M \rightarrow \lambda(1+\lambda)$ replacement discussed at the end of Appendix C, accounting approximately for relativistic kinematics in the energy-conserving $\delta$-function. As is clearly seen by comparing the solid and long-dashed curves, and in accord with past work [11, 12, 13], when both approximations are made the agreement is excellent, verifying that the use of the particular approximate currents and kinematics assumed here yields results that differ by only a few percent from the exact RFG results.

Also shown in Fig. 2 (as short-dashed curves) are responses having the relativized kinematics, but now using non-relativistic currents. In particular for these the factors $\kappa / \sqrt{\tau}$ and $\sqrt{\tau} / \kappa$ in Eqs. (20) and (22), respectively, are set to unity. Without the relativistic effects in the currents the results for $R^{L}\left(R^{T}\right)$ are too small (large), as expected. We note that conventional treatments 
of the single-nucleon current (see, for example, Eqs. (D4) and (D5) of Ref. [27]) often take $\omega \ll q$, in which case $\kappa \cong \sqrt{\tau}$. Moreover, as discussed in Appendix A, the non-relativistic expansions are made not only with respect to $\eta$, but also $\kappa, \lambda$ and $\tau$. Figure 2 demonstrates the magnitude of the error to be expected for QE scattering if one makes such approximations.

Finally in Fig. 2 are also shown results where the replacement $\lambda \rightarrow \lambda(1+\lambda)$ is not made (dot-dash curves with the relativistic corrections to the current, dotted curves without). With non-relativistic kinematics the QE peak occurs near $\omega=q^{2} / 2 M\left(\lambda=\kappa^{2}=\tau(1+\tau)\right)$, whereas with relativistic kinematics it occurs near $\omega=\left|Q^{2}\right| / 2 M(\lambda=\tau)$, namely, at a smaller value. As discussed in Ref. [10], the relativistic model yields a smaller width to the QE response than does the non-relativistic model. Also, the four-momentum transfer is decreasing as $\omega$ increases for fixed $q$ and consequently the single-nucleon form factors (those that are simply proportional to dipole form factors) increase with increasing $\omega$. This is the underlying reason for the large enhancements seen in the non-relativistic results shown in the figure.

The importance of maintaining relativistic kinematics in the energy-conserving $\delta$-function is obvious from these results. Indeed it would be a very poor approximation to ignore either of the "relativizing" steps made in the present work. Clearly as the momentum transfer reaches the medium-energy domain of interest in this work the effects of relativity in currents and kinematics (at least) are rather important. Typically one would incur significant error in using the completely non-relativistic FG model and thus, by extension, one must doubt any other model which does not incorporate the effects discussed in the present work. The CSM model discussed below has been developed with this in mind: we use only the relativized current operators and always make the replacement $\omega \rightarrow \omega(1+\omega / 2 M)$ in the energy-conserving $\delta$ functions. We now turn to the results obtained using that model.

\subsection{Quasielastic responses in the CSM}

An important aspect in the study of nuclear reactions is to determine as precisely as we can the nuclear physics dependences inherent in the observables. On the one hand, as in much of the present work, we may be interested in minimizing such dependences with the hope of extracting information about the nucleon's various form factors; on the other hand, it may be that certain observables contain unusually large sensitivity to some specific issue of interest in studying the nuclear many-body problem. The focus in the present work is largely to address the former of these two points of view specifically for the PV electron scattering observables. In this regard, the study of ratios of responses such as the PV asymmetry is favored, since they usually turn out to be less sensitive to the nature of the underlying dynamical assumptions made when compared with the response functions (or cross sections) themselves.

In particular, here we compare the results of calculations using the CSM with well parameters given in Table 1 with those of the RFG model for the response functions, the PV asymmetry 
and the sum-rule asymmetry, the last being defined below following previous studies in this area [13]. For the RFG results shown here we use as Fermi momenta the following: $p_{F}=$ 215 or $225 \mathrm{MeV} / \mathrm{c}$ for ${ }^{16} \mathrm{O}, 235$ or $245 \mathrm{MeV} / \mathrm{c}$ for ${ }^{40} \mathrm{Ca}$ and 230 or $250 \mathrm{MeV} / \mathrm{c}$ for protons in ${ }^{208} \mathrm{~Pb}$. In each case these have been chosen as representative for the three nuclei, with a smaller value that is favored somewhat when ground-state properties are emphasized and a larger value that yields rough agreement when the RFG model predictions are compared with experiment (this presumably arises since QE scattering at intermediate energies involves the ground state and a rather excited particle-hole state, both of which the model must recognize). For ${ }^{208} \mathrm{~Pb}$ we show results using two different assumptions for the relationship between the proton and neutron Fermi momenta, (1) with $p_{F n}=(N / Z)^{1 / 3} p_{F p}=265$ or $288 \mathrm{MeV} / \mathrm{c}$ for the two cases, corresponding to keeping the volume of the neutron and proton gases equal, and (2) with $p_{F n}=p_{F p}=250 \mathrm{MeV} / \mathrm{c}$, corresponding to equal densities for the two gases. As usual we take $p_{F n}=p_{F p}$ for ${ }^{16} \mathrm{O}$ and ${ }^{40} \mathrm{Ca}$. In the next subsection we show results for the PV asymmetry that indicate the very weak dependence of this observable on the Fermi momenta chosen, confirming past work [9].

The resulting EM and PV response functions are displayed in Figs. 3-8 for $q=300,500$ and $700 \mathrm{MeV} / \mathrm{c}$ (the PV results given here are shown in the absence of strangeness; see the later discussion in the next subsection). Specifically, in Fig. 3 we show the EM response functions for ${ }^{16} \mathrm{O}$, followed in Figs. 4-8 with results for the complete set of five electroweak inclusive responses for the cases of ${ }^{40} \mathrm{Ca}$ and ${ }^{208} \mathrm{~Pb}$. The inter-comparisons of $R^{L}$ and $R^{T}$ for oxygen and calcium are typical in that the full sets of responses in these two cases are very similar. Thus, for brevity we have focused on calcium and lead to show the nuclear model dependences obtained in going from light to heavy nuclei while omitting the PV responses for oxygen.

From the results in Figs. 3-8 we observe the following:

1. Overall, the RFG yields a reasonable description of the responses when the momentum transfer is high enough. The region where the RFG is Pauli-blocked (the $q=300 \mathrm{MeV} / \mathrm{c}$ case in the figures presented here) is where the agreement is the worst, as expected, since the RFG is known to become a poor approximation in this regime.

2. On closer examination, one sees that the CSM produces tails extending below and above the response region where the RFG is nonzero. Again, this is expected, since the latter model has a sharp cutoff to its momentum distribution while the former has all momentum components present, these being governed by the initial- and final-state wave functions used in the CSM. One also sees, in going from oxygen to calcium to lead at intermediateto-high values of $q$, that the agreement between the two models becomes better and better. The slight shift in peak position seen in Fig. 3 for oxygen is seen to go away for the heavier nuclei and the importance of the tails of the response functions produced by the CSM 
decreases with increasing $A$. With regard to the latter, in effect, the heavier the nucleus the more it appears to be dominated by volume effects, rather than surface effects, for the inclusive responses. At $q=300$ or $500 \mathrm{MeV} / \mathrm{c}$ and low energy we see somewhat irregular behaviour in the shell model responses which is a consequence of the contribution of the potential resonances in that region. This behaviour is more pronounced in the case of ${ }^{40} \mathrm{Ca}$ than for ${ }^{16} \mathrm{O}$, and, in the case of ${ }^{208} \mathrm{~Pb}$, it is responsible for the peak that clearly can be seen at $q=300 \mathrm{MeV} / \mathrm{c}$ and $\omega \cong 40 \mathrm{MeV}$ in both $R^{L}$ and $R^{T}$.

3. The rather good agreement between the RFG and CSM responses at high $q$ is only obtained when the approximations discussed above are made. If the currents and kinematics are not "relativized" as we have in this work, but instead are taken to be their usual nonrelativistic forms, then significant differences between the RFG and (conventional) CSM will be seen to occur.

4. When comparing the five different electroweak responses, the worst agreement between the models is seen for $R_{A V}^{L}$, although even there it is reasonably good for high $q$. It should be noted that this response is strongly suppressed in such mean-field calculations for the reasons discussed in Refs. [6, 9]; indeed, at $q=500 \mathrm{MeV} / \mathrm{c}$ for example, it is only a few percent of the leading PV response $R_{A V}^{T}$ (and the other, $R_{V A}^{T^{\prime}}$, is roughly $20 \%$ of this). As a consequence any differences seen here are unimportant for the PV asymmetry. On the other hand, especially at relatively low $q$ this is known to be only part of the full story. In particular, it has been argued [13] that correlation effects that fall outside the scope of the strict RFG and CSM approaches can strongly influence this particular response at low-to-moderate momentum transfers, at the same time only mildly effecting the other four response functions. In the next subsection we return to this issue when discussing the role played by the electric strangeness content of the nucleon.

5. In the figures we have shown the RFG results otained with the different choices for the Fermi momenta discussed above. The response functions are seen to differ somewhat, with a slight tendency to favor smaller values for $p_{F}$. As we shall see immediately below, even this mild dependence on the Fermi momenta is considerably lessened for the PV asymmetry.

Next we turn to the PV asymmetry. As seen in Fig. 9, such ratios are very insensitive to the choice of nuclear model made for the dynamics. Only in kinematic regions that correspond to the tails of the response functions do we see significant differences - and there the figure-of-merit for measuring the PV asymmetry is very small (see Ref. [6] for a discussion of the figure-ofmerit). As a consequence, we show only the regions where the figure-of-merit is appreciable in the figure. 
In the next subsection we shall discuss the sensitivity of PV observables to specific aspects of the nucleon's form factors. Following Ref. [13] we shall do this using the sum-rule asymmetry (SRA) defined as:

$$
\mathcal{R}\left(q, \theta_{e}\right) \equiv \frac{\int_{0}^{\infty} d \omega W_{\mathrm{pv}}\left(q, \omega, \theta_{e}\right) / \tilde{X}_{T}^{\prime}}{\int_{0}^{\infty} d \omega W_{\mathrm{em}}\left(q, \omega, \theta_{e}\right) / X_{T}^{\prime}},
$$

where the functions $X_{T}^{\prime}$ and $\tilde{X}_{T}^{\prime}$ are given in Ref. 113. These functions depend on the Fermi momentum and for simplicity we have used only the values (in $\mathrm{MeV} / \mathrm{c}) p_{F}=225\left({ }^{16} \mathrm{O}\right), 235$ $\left({ }^{40} \mathrm{Ca}\right)$ and $250 / 288\left(\mathrm{p} / \mathrm{n}{ }^{208} \mathrm{~Pb}\right)$. The dependence on $p_{F}$ here is negligible and so choosing any reasonable value is an excellent approximation. The motivation for using the specific derived quantity $\mathcal{R}$ is that it has been designed to be effective in removing most of the rapid variation of the single-nucleon form factors, to the extent that this is possible. While constructed with the RFG in mind, however, it should be noted that it merely leads to a reduced quantity in much the same way that dividing the nuclear cross section by the Mott cross section leads to a form factor - there is no loss of information, only a re-expression of the same information. Experimentally this quantity can be constructed from the measured helicity-difference and helicity-sum cross sections (PV and EM, respectively) upon dividing by the $X$ 's and integrating over a range in $\omega$. For the RFG the range of integration is naturally taken to include the entire response, since the sharp cutoff guarantees specific limits. In the case of the CSM, we integrate from $\omega$ at threshold up to sufficiently large values of $\omega$ that the integrals in the numerator and denominator of Eq. (57) saturate.

In Fig. 10 we show the SRA for ${ }^{40} \mathrm{Ca}$ and ${ }^{208} \mathrm{~Pb}$ (the ${ }^{16} \mathrm{O}$ results are very similar to the former and so are not shown). The sets of curves shown are described in the figure captions (see Fig. 4 for the basic key). Rather clearly the nuclear model dependence is very weak, especially for intermediate-to-high values of $q$, in that the various curves cannot be distinguished in the figure. This weak nuclear model dependence is essential if one wishes to use nuclei to explore properties of the nucleon itself.

In Fig. 11 we show the CSM predictions for the SRA now for all three nuclei, observing the near universality of this observable for a large range of nuclei with only slight differences appearing when lead is compared with oxygen and calcium (the last two are essentially indistinguishable).

\subsection{Sensitivity to variations in the form factors of the nucleon}

We now turn to an assessment of the nuclear model dependences seen using the CSM and RFG when attempting to extract information about the isovector axial-vector and electric strangeness form factors of the nucleon. We do this using the sum-rule asymmetry defined above.

We begin by presenting results in Fig. 12 for the SRA for ${ }^{40} \mathrm{Ca}$ and ${ }^{208} \mathrm{~Pb}$ with no magnetic strangeness (as in the previous figures) and with $\mu_{s}$ in Eq. (38) set equal to -0.35 . The latter 
is chosen in accordance with the discussions of modeling the nucleon's strangeness (see [6] and references therein). For comparison, as also discussed in that reference, we note that the SAMPLE experiment being performed at Bates to measure backward-angle PV $\overrightarrow{e p}$ scattering is expected to have an uncertainty of approximately $\left|\Delta \mu_{s}\right| \sim 0.2$. In the figure we see that the nuclear model dependence is weaker than the dependence on $G_{M}^{(s)}$ for intermediate-to-high values of $q$ in the case of an $N=Z$ nucleus such as ${ }^{40} \mathrm{Ca}\left({ }^{16} \mathrm{O}\right.$ is almost indistinguishable from ${ }^{40} \mathrm{Ca}$ ). However, at low $q$ or for nuclei with $N$ significantly larger than $Z$, such as ${ }^{208} \mathrm{~Pb}$, the $G_{M}^{(s)}$ dependence is much weaker. As discussed in Ref. [9], this arises from the fact that in the $\mathrm{PV}$ asymmetry this form factor is multiplied by the combination $\left(Z G_{M}^{p}+N G_{M}^{n}\right) / A$, which is proportional to $\left(Z \mu_{p}+N \mu_{n}\right) / A=0.44(-0.06)$ for calcium (lead).

For backward-angle PV QE electron scattering the asymmetry depends importantly on the magnetic strangeness form factor $G_{M}^{(s)}$ discussed above and the isovector, axial-vector form factor $G_{A}^{(T=1)}$ parametrized in Eq. (36). The electric strangeness form factor $G_{E}^{(s)}$ is unimportant for $\theta_{e}$ large. In Fig. 13 we show the dependence on $G_{A}^{(T=1)}$. The three sets of curves shown correspond to results with the isovector, axial-vector coupling at $Q^{2}=0$ taken to be its canonical value, $g_{A}=1.262$, together with results when $g_{A}=1.262 \pm 10 \%$ (indicated with a $( \pm)$ in the figure). In all cases the strangeness content of the nucleon has been neglected. Clearly, as in Ref. [13 where other nuclear models were inter-compared in the same way, for intermediate values of momentum transfer the nuclear model dependences are quite weak compared with the level of variation in $\mathcal{R}$ caused by $10 \%$ changes in the isovector axial-vector coupling. Hence the conclusion found in previous work still stands that measurements of this observable could constrain the NC axialvector form factor of the nucleon with interesting precision. It is also clear from Figs. 12 and 13 that measurements on two nuclei are needed to determine both $\mu_{s}$ and $g_{A}-\mathrm{PV}$ elastic scattering from the proton and PV QE scattering from nuclei such as those discussed in the present work or the deuteron discussed in Ref. [28] would provide the required information.

Turning finally to a discussion of forward-angle PV QE electron scattering we focus on the dependences in the SRA on $G_{E}^{n}, G_{M}^{(s)}$ and $G_{E}^{(s)}\left(G_{A}^{(T=1)}\right.$ is unimportant for $\theta_{e}$ small). In Fig. 14 $\mathcal{R}$ is shown for three values of the Galster parameter $\lambda_{n}$ in Eq. (34), corresponding to $\pm 10 \%$ variation in $G_{E}^{n}$ at its peak value. This sets a scale for the uncertainty expected in the SRA at forward angles, being typical of the hoped-for precision in determining $G_{E}^{n}$ from polarization measurements of parity-conserving $e^{2} \mathrm{H}$ and $e^{3} \mathrm{He}$ scattering. The $G_{M}^{(s)}$ sensitivity has been shown in Fig. 12 and the dependence on $G_{E}^{(s)}$ is shown in Fig. 15. In the latter three models are employed: (I) no electric strangeness; (II) $G_{E}^{(s)}$ with $\rho_{s}=-3, \lambda_{E}^{(s)}=5.6$ in Eq. (37); and (III) $G_{E}^{(s)}$ with $\rho_{s}=-3, \lambda_{E}^{(s)}=0$. Model (II) corresponds to "modest electric strangeness", while model (III) has "large electric strangeness" (see Ref. [6]). Clearly when the interest is to see effects from electric strangeness the momentum transfer cannot be too low, since the single-nucleon form factor $G_{E}^{(s)}$ vanishes as $\left|Q^{2}\right| \rightarrow 0$ (see Eq. (37)). In the figure we have chosen 
to present results for ${ }^{40} \mathrm{Ca}$ and ${ }^{208} \mathrm{~Pb}$ at $q=300,500$ and $700 \mathrm{MeV} / \mathrm{c}$; again the ${ }^{16} \mathrm{O}$ and ${ }^{40} \mathrm{Ca}$ results are rather similar and so the former are not displayed. We see that at forward angles and high momentum transfer the sum-rule weighted asymmetry shows clear signatures of the electric strangeness, while at lower values of $q$ the nuclear model dependences become important enough for the case of ${ }^{208} \mathrm{~Pb}$ to obscure the $G_{E}^{(s)}$ effects. Comparing Figs. 12,14 and 15 it is clear that, given PV $\overrightarrow{e p}$ and QE information at backward angles to determine $G_{M}^{(s)}$ and $G_{A}^{(T=1)}$ as well as possible, forward-angle PV QE scattering at high $q$ should be capable of determining

$G_{E}^{(s)}$. For low $q$ the situation is much less clear. For ${ }^{40} \mathrm{Ca}$ the results in Fig. 15 would appear to indicate at least that models II and III could be distinguished from model I for the electric strangeness. However, it is already clear from the results presented in Ref. 13] that correlations beyond the mean field play a dominant role at low $q$. In recent work [29] these effects have been explored in depth and, while the determining factor in the SRA at forward angles and low- $q$, they become sufficiently weak at intermediate-to-high values of $q$ that the above conclusions concerning the dominant sensitivity to $G_{E}^{(s)}$ remain valid.

\section{Conclusions}

In conclusion, in this work we have (1) developed the single-nucleon vector and axial-vector current operators for use in parity-conserving and -violating electron scattering studies, (2) explored approximations to these currents and to specific aspects of relativistic kinematics within the context of the Fermi gas model, and (3) applied the same ideas to studies of electroweak QE responses and asymmetries in the continuum shell model.

In developing the current operators we have seen that the traditional expansions in all dimensionless momenta provide poor approximations to the correct on-shell answers at high momentum transfers. By making more limited expansions in $\eta=p / M$, but not in $\kappa=q / 2 M$, $\lambda=\omega / 2 M$ or $\tau=\kappa^{2}-\lambda^{2}$ we have obtained approximate currents that should be appropriate in the region where the QE responses are large. Naturally, if $\eta$ is of order unity or large, then this scheme will be suspect; however, in such circumstances the idea of using any on-shell current must also be questioned and we must resort to off-shell prescriptions for the currents.

To evaluate the quality of the approximations made to the currents we have considered the relativistic Fermi gas model where exact (model) results exist. We have verified that the approximate currents are very successful for the closed shell (spin-saturated) nuclei studied in this work. Furthermore, we have verified that the non-relativistic Fermi gas model extended by incorporating the approximate currents and as well by "relativizing" the kinematics provides excellent agreement with the RFG results for the electroweak responses. This has led us to propose that the same approximate currents and assumption about the kinematics be invoked for any non-relativistic model of the nuclear dynamics involved in QE electron scattering. 
In particular, we have made these assumptions in computing the QE responses with the continuum shell model. The results are very encouraging: the resulting electroweak response functions are rather similar to those found with the RFG model when the momentum transfer is high enough for the latter to be valid. Said the opposite way, if the two assumptions concerning the currents and kinematics were not to be made then the CSM and RFG results would be dramatically different at high $q$.

Finally, we have employed the RFG (with various choices of Fermi momenta) and the CSM to study the PV asymmetry. We find very little nuclear model dependence in the results, especially those relating to the sum-rule asymmetry. This confirms the belief from other work that at high $q$ the SRA can be used to study specific properties of the nucleon itself without incurring too much uncertainty from the nuclear modeling. Backward-angle PV QE scattering together with PV elastic $\overrightarrow{e p}$ scattering will serve to determine the magnetic strangeness and isovector, axialvector form factors of the nucleon, while forward-angle PV QE scattering at high- $q$ may add information about the electric strangeness form factor of the nucleon.

\section{Appendix A: On-Shell Single-Nucleon Electroweak Current Operators}

The on-shell single-nucleon four-vector EM current may be written in the form:

$$
J^{\mu}\left(P \Lambda ; P^{\prime} \Lambda^{\prime}\right)=\bar{u}\left(P^{\prime} \Lambda^{\prime}\right)\left[F_{1} \gamma^{\mu}+\frac{i}{2 M} F_{2} \sigma^{\mu \nu} Q_{\nu}\right] u(P \Lambda),
$$

where the incident nucleon has four-momentum $P^{\mu}=(E, \boldsymbol{p})$, the outgoing nucleon has fourmomentum $P^{\prime \mu}=\left(E^{\prime}, \boldsymbol{p}^{\prime}\right)$ and $Q^{\mu}=P^{\prime \mu}-P^{\mu}$. The spin projections are labeled $\Lambda$ and $\Lambda^{\prime}$ for incoming and outgoing nucleons, respectively. As in previous work [10], the dimensionless variables

$$
\begin{aligned}
\lambda & \equiv \omega / 2 M \\
\boldsymbol{\kappa} & \equiv \boldsymbol{q} / 2 M \\
\tau & \equiv \kappa^{2}-\lambda^{2} \\
\boldsymbol{\eta} & \equiv \boldsymbol{p} / M \\
\varepsilon & \equiv E / M=\sqrt{1+\eta^{2}}
\end{aligned}
$$

prove to be convenient. Let us also introduce the angle $\theta$ between $\boldsymbol{\kappa}$ and $\boldsymbol{\eta}$. We then have the following relationships amongst the kinematic variables:

$$
\begin{aligned}
\kappa \eta \cos \theta & =\lambda \varepsilon-\tau \\
\tau(\varepsilon+\lambda)^{2} & =\kappa^{2}\left(1+\tau+\delta^{2}\right),
\end{aligned}
$$


where we define $\delta \equiv \eta \sin \theta$. For reasons that will become apparent later we also define the following quantities:

$$
\begin{aligned}
\mu_{1} & \equiv \frac{\kappa \sqrt{1+\tau}}{\sqrt{\tau}(\varepsilon+\lambda)}=\frac{1}{\sqrt{1+\frac{1}{1+\tau} \delta^{2}}} \\
\mu_{2} & \equiv \frac{2 \kappa \sqrt{1+\tau}}{\sqrt{\tau}(1+\tau+\varepsilon+\lambda)}=\frac{2 \mu_{1}}{1+\frac{\sqrt{\tau(1+\tau)}}{\kappa} \mu_{1}} .
\end{aligned}
$$

To evaluate the current in Eq. (58) we insert the appropriate $\gamma$-matrices between the spinors

$$
\begin{aligned}
\bar{u}\left(P^{\prime} \Lambda^{\prime}\right) & =\frac{1}{\sqrt{2}} \sqrt{1+\varepsilon^{\prime}} \chi_{\Lambda^{\prime}}^{\dagger}\left(1,-\frac{1}{1+\varepsilon^{\prime}} \boldsymbol{\eta}^{\prime} \cdot \boldsymbol{\sigma}\right) \\
u(P \Lambda) & =\frac{1}{\sqrt{2}} \sqrt{1+\varepsilon}\left(\begin{array}{c}
1 \\
\frac{1}{1+\varepsilon} \boldsymbol{\eta} \cdot \boldsymbol{\sigma}
\end{array}\right) \chi_{\Lambda},
\end{aligned}
$$

where $\chi_{\Lambda}$ and $\chi_{\Lambda^{\prime}}$ are the usual 2-component spin- $\frac{1}{2}$ spinors, with $\boldsymbol{\eta}^{\prime}$ and $\varepsilon^{\prime}$ the final outgoing nucleon analogs of the unprimed quantities. We wish to have expressions for the single-nucleon EM current operators $\bar{J}^{\mu}\left(P ; P^{\prime}\right)$ that occur inside these latter quantities, viz.

$$
J^{\mu}\left(P \Lambda ; P^{\prime} \Lambda^{\prime}\right) \equiv \chi_{\Lambda^{\prime}}^{\dagger} \bar{J}^{\mu}\left(P ; P^{\prime}\right) \chi_{\Lambda} .
$$

We use the bar over the current in the present appendix to distinguish an operator from its spin matrix elements; in the body of the paper for simplicity we suppress this notation. Writing these in the following way with an overall factor $f_{0}$ removed (note that $V^{\mu}$ is not a four-vector),

$$
\begin{aligned}
\bar{J}^{\mu} & \equiv f_{0} V^{\mu} \\
f_{0} & \equiv \frac{1}{\mu_{1} \sqrt{1+\frac{\tau}{4(1+\tau)} \mu_{2}^{2} \delta^{2}}}
\end{aligned}
$$

the EM current operator may then be expressed in terms of the quantities defined above,

$$
\begin{aligned}
V^{0}= & \xi_{0}+i \xi_{0}^{\prime}(\boldsymbol{\kappa} \times \boldsymbol{\eta}) \cdot \boldsymbol{\sigma} \\
V^{3}= & (\lambda / \kappa) V^{0} \\
\boldsymbol{v}^{\perp}= & \xi_{1}\left[\boldsymbol{\eta}-\left(\frac{\boldsymbol{\kappa} \cdot \boldsymbol{\eta}}{\kappa^{2}}\right) \boldsymbol{\kappa}\right]-i\left\{\xi_{1}^{\prime}(\boldsymbol{\kappa} \times \boldsymbol{\sigma})\right. \\
& \left.+\xi_{2}^{\prime}(\boldsymbol{\kappa} \cdot \boldsymbol{\sigma})(\boldsymbol{\kappa} \times \boldsymbol{\eta})+\xi_{3}^{\prime}[(\boldsymbol{\kappa} \times \boldsymbol{\eta}) \cdot \boldsymbol{\sigma}]\left[\boldsymbol{\eta}-\left(\frac{\boldsymbol{\kappa} \cdot \boldsymbol{\eta}}{\kappa^{2}}\right) \boldsymbol{\kappa}\right]\right\},
\end{aligned}
$$

where the $\xi$ 's (no spin dependence) and $\xi^{\prime \prime s}$ (spin dependence) are the following:

$$
\xi_{0}=\frac{\kappa}{\sqrt{\tau}}\left[G_{E}+\frac{\mu_{1} \mu_{2}}{2(1+\tau)} \delta^{2} \tau G_{M}\right]
$$




$$
\begin{aligned}
\xi_{0}^{\prime} & =\frac{1}{\sqrt{1+\tau}}\left[\mu_{1} G_{M}-\frac{1}{2} \mu_{2} G_{E}\right] \\
\xi_{1} & =\frac{1}{\sqrt{1+\tau}}\left[\mu_{1} G_{E}+\frac{1}{2} \mu_{2} \tau G_{M}\right] \\
\xi_{1}^{\prime} & =\frac{\sqrt{\tau}}{\kappa}\left(1-\frac{\mu_{1} \mu_{2}}{2(1+\tau)} \delta^{2}\right) G_{M} \\
\xi_{2}^{\prime} & =\frac{\sqrt{\tau}}{2 \kappa^{2} \sqrt{1+\tau}} \sqrt{\tau \mu_{2}^{2}+4 \mu_{1}\left(\mu_{2}-\mu_{1}\right)-\left(\mu_{1} \mu_{2} \delta\right)^{2}} G_{M} \\
& =\frac{\lambda \sqrt{\tau}}{2 \kappa^{3}} \mu_{1} \mu_{2} G_{M} \\
\xi_{3}^{\prime} & =\frac{\sqrt{\tau}}{2 \kappa(1+\tau)} \mu_{1} \mu_{2}\left[G_{E}-G_{M}\right] .
\end{aligned}
$$

These expressions constitute exact expressions for the on-shell EM current operator. Equation (68) reflects the fact that the current is conserved.

The vector $\mathrm{NC}$ analogs of the results here are simply obtained by replacing $G_{E}$ by $\tilde{G}_{E}$ and $G_{M}$ by $\tilde{G}_{M}$.

The NC axial-vector current may be written as in Eq. (58):

$$
J_{5}^{\mu}\left(P \Lambda ; P^{\prime} \Lambda^{\prime}\right)=\bar{u}\left(P^{\prime} \Lambda^{\prime}\right)\left[\tilde{G}_{A} \gamma^{\mu}+\frac{i}{2 M} \tilde{G}_{P} Q^{\mu}\right] \gamma_{5} u(P \Lambda)
$$

where the " 5 " is used to indicate axial-vector quantities (from the extra $\gamma_{5}$ above, compared with the EM current in Eq. (58)) and where $\tilde{G}_{A}$ and $\tilde{G}_{P}$ are the axial and induced pseudoscalar form factors of the nucleon, respectively. The analog of Eq. (64) becomes

$$
J_{5}^{\mu}\left(P \Lambda ; P^{\prime} \Lambda^{\prime}\right) \equiv \chi_{\Lambda^{\prime}}^{\dagger} \bar{J}_{5}^{\mu}\left(P ; P^{\prime}\right) \chi_{\Lambda}
$$

with

$$
\bar{J}_{5}^{\mu} \equiv f_{0} A^{\mu}
$$

where $f_{0}$ is given in Eq. (66) (as above, here $A^{\mu}$ is not a four-vector). Clearly the pseudoscalar contributions are absent for transverse ( 1 and 2 components) projections of the current, since the coordinate system used has been chosen to have the momentum transfer along the 3-direction. One finds from carrying out the procedures discussed above for the EM (vector) current, inserting the spinors and $\gamma$-matrices, that the transverse projections are given by

$$
\boldsymbol{a}^{\perp}=\left\{\zeta_{1}^{\prime} \boldsymbol{\sigma}^{\perp}+\zeta_{2}^{\prime}[(\boldsymbol{\kappa}+\boldsymbol{\eta}) \cdot \boldsymbol{\sigma}]\left[\boldsymbol{\eta}-\left(\frac{\boldsymbol{\kappa} \cdot \boldsymbol{\eta}}{\kappa^{2}}\right) \boldsymbol{\kappa}\right]-i \zeta_{1}(\boldsymbol{\kappa} \times \boldsymbol{\eta})\right\} \tilde{G}_{A}
$$

with

$$
\begin{aligned}
\zeta_{1} & =\zeta_{2}^{\prime}=\frac{1}{2}\left(\frac{\sqrt{\tau}}{\kappa}\right) \mu_{1} \mu_{2} \\
\zeta_{1}^{\prime} & =\sqrt{1+\tau} \mu_{1},
\end{aligned}
$$


in parallel with Eqs. (70).

The exact results given above in Eqs. (64)-(70) may be used in treating EM interactions with an on-shell nucleon. However, for applications to nuclear physics as in the present work where nucleons are off-shell in general it is necessary to make some approximations. What is often done is to continue to use the above expressions as operators inserted between nuclear wave functions, where the latter are not on-shell plane waves but are the single-particle wave functions for nucleons in nuclei (i.e., in the presence of the nuclear mean field). The coordinate space operator corresponding to $\boldsymbol{\eta}$ is then $-\frac{i}{M} \boldsymbol{\nabla}$. For typical situations the nucleon to which the virtual photon attaches is relatively low in energy — the dimensionless momentum $\eta \sim \eta_{F}=p_{F} / M \sim 0.25$ yielding dimensionless energy $\varepsilon \cong 1+\frac{1}{2} \eta^{2} \sim 1+\frac{1}{2} \eta_{F}^{2} \sim 1$.03. Accordingly the usual practice is to expand the expressions for the EM current given above (at least) in powers of $\eta$, retaining only the leading-order terms. Equations (70,75) have been cast in forms that make this easy. Let us work to linear order in $\eta$. First, from Eqs. (61) and (66) we have $\mu_{1}=1+\mathcal{O}\left(\eta^{2}\right)$ and $f_{0}=1+\mathcal{O}\left(\eta^{2}\right)$, whereas it may be shown that $\mu_{2}=1+\frac{1}{2} \sqrt{\frac{\tau}{1+\tau}} \eta \cos \theta+\mathcal{O}\left(\eta^{2}\right)$ by using Eqs. 60 62). We then have for the EM current operators carried to order $\eta$ :

$$
\begin{aligned}
\bar{J}^{0}= & \frac{\kappa}{\sqrt{\tau}} G_{E}+\frac{i}{\sqrt{1+\tau}}\left[G_{M}-\frac{1}{2} G_{E}\right](\boldsymbol{\kappa} \times \boldsymbol{\eta}) \cdot \boldsymbol{\sigma}+\mathcal{O}\left(\eta^{2}\right) \\
\bar{J}^{3}= & (\lambda / \kappa) \bar{J}^{0} \\
\overline{\boldsymbol{J}}^{\perp}= & -\frac{\sqrt{\tau}}{\kappa}\left\{i G_{M}\left([\boldsymbol{\kappa} \times \boldsymbol{\sigma}]+\frac{1}{2(1+\tau)}(\boldsymbol{\kappa} \cdot \boldsymbol{\sigma})(\boldsymbol{\kappa} \times \boldsymbol{\eta})\right)\right. \\
& \left.-\left(G_{E}+\frac{1}{2} \tau G_{M}\right)\left[\boldsymbol{\eta}-\left(\frac{\boldsymbol{\kappa} \cdot \boldsymbol{\eta}}{\kappa^{2}}\right) \boldsymbol{\kappa}\right]\right\}+\mathcal{O}\left(\eta^{2}\right),
\end{aligned}
$$

employing Eqs. (67 69) and noting from Eq. (60) that, after some work, $\kappa=\sqrt{\tau(1+\tau)}+$ $\tau \eta \cos \theta+\mathcal{O}\left(\eta^{2}\right)$. Of course, when computing matrix elements of these operators and then forming bilinear combinations of the results to obtain the EM observables terms of order $\eta^{2}$ must be neglected if the operators themselves have been expanded only to order $\eta$, since other terms will enter from considering the neglected $\mathcal{O}\left(\eta^{2}\right)$ contributions in Eqs. (76 78).

Note that we have only expanded in powers of $\eta$, having argued that this is typically a small quantity. Indeed, if it is not small (for instance, when considering very high momentum components in the nuclear wave function, viz. $\eta \sim 1 \leftrightarrow p \sim M)$ then the full expressions must be employed; however, in such circumstances the struck nucleon is very far off-shell and the entire procedure becomes doubtful. What we have not done is to expand in powers of the other dimensionless momenta, $\kappa, \lambda$ or $\tau$. To see why this can be a problem at moderate momentum transfers note that the typical observables that occur (for example, $W_{2}=\left(G_{E}^{2}+\tau G_{M}^{2}\right) /(1+\tau)$ ) involve combinations of the form factors where one often has $G_{E}^{2}$ together with $\left(\sqrt{\tau} G_{M}\right)^{2}$. Using the dipole approximation to estimate the size of these quantities we see that they become equal for protons at $\tau \cong 0.13 \leftrightarrow\left|Q^{2}\right| \cong(670 \mathrm{MeV} / \mathrm{c})^{2}$, for neutrons at $\tau \cong 0.27 \leftrightarrow\left|Q^{2}\right| \cong(980$ 
$\mathrm{MeV} / \mathrm{c})^{2}$, or alternatively, for isovector contributions at $\tau \cong 0.045 \leftrightarrow\left|Q^{2}\right| \cong(400 \mathrm{MeV} / \mathrm{c})^{2}$ and for isoscalar contributions at $\tau \cong 1.29 \leftrightarrow\left|Q^{2}\right| \cong(2.1 \mathrm{GeV} / \mathrm{c})^{2}$. Clearly for many practical applications it is not valid only to retain terms of leading order in $\sqrt{\tau}$ or $\kappa$. Moreover, as we see from the results presented in Sect. 3.1, the distinction between $\kappa$ and $\sqrt{\tau}$ becomes important for QE scattering at high momentum transfer. In fact, as we see from the expressions given above, it is unnecessary to make expansions in $\kappa, \lambda$ or $\tau$ at all. If one does, however, choose to do so, then at intermediate momentum transfers the combination $G_{M}^{\prime} \equiv \sqrt{\tau} G_{M}$ should be regarded as being of leading order, and not of order $M^{-2}$ as is often assumed. With these caveats under some circumstances Eq. (78) may be approximated by

$$
\overline{\boldsymbol{J}}^{\perp} \cong-\frac{\sqrt{\tau}}{\kappa}\left\{i G_{M}[\boldsymbol{\kappa} \times \boldsymbol{\sigma}]-G_{E}\left[\boldsymbol{\eta}-\left(\frac{\boldsymbol{\kappa} \cdot \boldsymbol{\eta}}{\kappa^{2}}\right) \boldsymbol{\kappa}\right]\right\} .
$$

At this point it should be mentioned that neglecting the second term in Eq. (76) (the spinorbit term), which is order $\kappa \eta$ compared with the first term which is of order unity, may not always be justified. However, in spin-saturated systems such as the closed-shell nuclei considered in the present work this term contributes only quadratically and may safely be neglected when computing EM responses. For instance, the contribution of the spin-orbit term to $R^{L}$ and $R_{A V}^{L}$ in a spin-saturated system can be estimated using the RFG model to be $\frac{3}{10} \eta_{F}^{2} \frac{\tau}{1+\tau}\left(1+\frac{G_{M}^{2}}{G_{E}^{2}}\right)$ and $\frac{3}{10} \eta_{F}^{2} \frac{\tau}{1+\tau}\left(1+\frac{G_{M} \tilde{G}_{M}}{G_{E} \tilde{G}_{E}}\right)$, respectively, compared with the leading-order contributions $\frac{\kappa^{2}}{\tau} G_{E}^{2}$ and $\frac{\kappa^{2}}{\tau} G_{E} \tilde{G}_{E}$. For typical values of $\eta_{F}\left(\sim \frac{1}{4}\right)$ the correction to $R^{L}$ is negligible $(<4 \%)$, while the correction to $R_{A V}^{L}$ is sizable $(\sim 40 \%)$ and has been taken into account in the results presented in Sect. 3. There we see that the latter response is quite small compared with "normal" response functions and thus this correction is relatively unimportant.

As before, the vector NC analogs of these EM results are obtained simply by making the replacements $G_{E, M} \rightarrow \tilde{G}_{E, M}$. Likewise, the axial-vector results above may be expanded in powers of $\eta$ following the procedures used for the EM case, yielding the transverse axial-vector current operator to order $\eta$ :

$$
\begin{aligned}
\overline{\boldsymbol{J}}_{5}^{\perp}= & \sqrt{1+\tau} \tilde{G}_{A}\left\{\boldsymbol{\sigma}^{\perp}\right. \\
& \left.+\frac{1}{2(1+\tau)}\left((\boldsymbol{\kappa} \cdot \boldsymbol{\sigma})\left[\boldsymbol{\eta}-\left(\frac{\boldsymbol{\kappa} \cdot \boldsymbol{\eta}}{\kappa^{2}}\right) \boldsymbol{\kappa}\right]-i(\boldsymbol{\kappa} \times \boldsymbol{\eta})\right)\right\} \\
\cong & \sqrt{1+\tau} \tilde{G}_{A} \boldsymbol{\sigma}^{\perp}
\end{aligned}
$$

where in Eq. (81) the second term, involving corrections of order $\kappa \eta$ compared with the first term, has been neglected.

Finally, in the discussions in the main body of the paper we also refer to the strict nonrelativistic limit. In the present work we take this to mean that the currents in Eqs. (76, 79, 81) are futher approximated by taking $\kappa$ and $\tau$ to be small compared with unity to yield

$$
\bar{J}^{0} \longrightarrow G_{E}
$$




$$
\begin{aligned}
& \overline{\boldsymbol{J}}^{\perp} \longrightarrow-i G_{M}[\boldsymbol{\kappa} \times \boldsymbol{\sigma}]+G_{E}\left[\boldsymbol{\eta}-\left(\frac{\boldsymbol{\kappa} \cdot \boldsymbol{\eta}}{\kappa^{2}}\right) \boldsymbol{\kappa}\right] \\
& \overline{\boldsymbol{J}}_{5}^{\perp} \longrightarrow \tilde{G}_{A} \boldsymbol{\sigma}^{\perp} .
\end{aligned}
$$

\section{Appendix B: Reduced Matrix Elements in the Shell Model}

After a laborious but straightforward calculation involving angular momentum algebra, the different reduced matrix elements involved in the PV responses can be written as follows: for the EM matrix elements one has

$$
\begin{aligned}
& \left\langle p h^{-1}, J\left\|M_{\mathrm{em}}^{J}\right\| 0\right\rangle=\frac{(-1)^{j_{p}-1 / 2}}{\sqrt{4 \pi}} \frac{\kappa}{\sqrt{\tau}} G_{E} \xi\left(\ell_{p}+\ell_{h}+J\right) \hat{j}_{p} \hat{j}_{h} \hat{J}\left(\begin{array}{ccc}
j_{p} & j_{h} & J \\
1 / 2 & -1 / 2 & 0
\end{array}\right) \\
& \times \int_{0}^{\infty} d r r^{2} j_{J}(q r) R_{p}(r) R_{h}(r) \\
& \left\langle p h^{-1}, J\left\|T_{\mathrm{em}}^{E J}\right\| 0\right\rangle_{\mathrm{mag}}=\frac{(-1)^{j_{p}+1 / 2}}{\sqrt{4 \pi J(J+1)}} \sqrt{\tau} G_{M} \xi\left(\ell_{p}+\ell_{h}+J\right) \hat{j}_{p} \hat{j}_{h} \hat{J}\left(\begin{array}{ccc}
j_{p} & j_{h} & J \\
1 / 2 & -1 / 2 & 0
\end{array}\right) \\
& \times\left(\gamma_{p}-\gamma_{h}\right) \int_{0}^{\infty} d r r^{2} j_{J}(q r) R_{p}(r) R_{h}(r) \\
& \left\langle p h^{-1}, J\left\|T_{\mathrm{em}}^{E J}\right\| 0\right\rangle_{\mathrm{conv}}=\frac{(-1)^{j_{p}-1 / 2}}{\sqrt{4 \pi J(J+1)}} \frac{\sqrt{\tau}}{\kappa} G_{E} \xi\left(\ell_{p}+\ell_{h}+J\right) \hat{j}_{p} \hat{j}_{h} \hat{J}\left(\begin{array}{ccc}
j_{p} & j_{h} & J \\
1 / 2 & -1 / 2 & 0
\end{array}\right) \\
& \times \frac{1}{2 M} \int_{0}^{\infty} d r r j_{J}(q r) \frac{1}{q}\left\{\left[\left(\gamma_{p}-\gamma_{h}\right)\left(\gamma_{p}+\gamma_{h}+1\right)+J(J+1)\right] R_{p}(r) \frac{d}{d r} R_{h}(r)\right. \\
& \left.+\left[\left(\gamma_{p}-\gamma_{h}\right)\left(\gamma_{p}+\gamma_{h}+1\right)-J(J+1)\right] R_{h}(r) \frac{d}{d r} R_{p}(r)\right\} \\
& \left\langle p h^{-1}, J\left\|T_{\mathrm{em}}^{M J}\right\| 0\right\rangle_{\mathrm{mag}}=\frac{i(-1)^{j_{p}-1 / 2}}{\sqrt{4 \pi J(J+1)}} \sqrt{\tau} G_{M} \xi\left(\ell_{p}+\ell_{h}+J+1\right) \hat{j}_{p} \hat{j}_{h} \hat{J} \\
& \times\left(\begin{array}{ccc}
j_{p} & j_{h} & J \\
1 / 2 & -1 / 2 & 0
\end{array}\right) \int_{0}^{\infty} d r r^{2} j_{J}(q r) \frac{1}{q}\left[\left(\gamma_{p}+\gamma_{h}\right)\left(\frac{d}{d r}+\frac{1}{r}\right)+\frac{J(J+1)}{r}\right] R_{p}(r) R_{h}(r) \\
& \left\langle p h^{-1}, J\left\|T_{\mathrm{em}}^{M J}\right\| 0\right\rangle_{\mathrm{conv}}=\frac{i(-1)^{j_{p}-1 / 2}}{\sqrt{4 \pi J(J+1)}} \frac{\sqrt{\tau}}{\kappa} G_{E} \xi\left(\ell_{p}+\ell_{h}+J+1\right) \hat{j}_{p} \hat{j}_{h} \hat{J} \\
& \times\left(\begin{array}{ccc}
j_{p} & j_{h} & J \\
1 / 2 & -1 / 2 & 0
\end{array}\right)\left[\left(\gamma_{p}+\gamma_{h}\right)\left(\gamma_{p}+\gamma_{h}+1\right)-J(J+1)\right] \frac{1}{2 M} \int_{0}^{\infty} d r r j_{J}(q r) R_{p}(r) R_{h}(r),
\end{aligned}
$$


together with their vector NC analogs, obtained by replacing $G_{E}$ with $\tilde{G}_{E}$ and $G_{M}$ with $\tilde{G}_{M}$. For the axial-vector $\mathrm{NC}$ operators, using the relations given by Eqs. (54,55), one simply obtains

$$
\begin{aligned}
&\left\langle p h^{-1}, J\left\|\left(T_{\mathrm{nc}}^{E J}\right)_{A}\right\| 0\right\rangle=\frac{i(-1)^{j_{p}-1 / 2}}{\sqrt{4 \pi J(J+1)}} \sqrt{1+\tau} \tilde{G}_{A} \xi\left(\ell_{p}+\ell_{h}+J+1\right) \hat{j}_{p} \hat{j}_{h} \hat{J} \\
& \times\left(\begin{array}{ccc}
j_{p} & j_{h} & J \\
1 / 2 & -1 / 2 & 0
\end{array}\right) \int_{0}^{\infty} d r r^{2} j_{J}(q r) \frac{1}{q}\left[\left(\gamma_{p}+\gamma_{h}\right)\left(\frac{d}{d r}+\frac{1}{r}\right)+\frac{J(J+1)}{r}\right] R_{p}(r) R_{h}(r) \\
&\left\langle p h^{-1}, J\left\|\left(T_{\mathrm{nc}}^{M J}\right)_{A}\right\| 0\right\rangle= \\
& \times\left(\gamma_{p}-\gamma_{h}\right) \int_{0}^{\infty} d r r^{2} j_{J}(q r) R_{p}(r) R_{h}(r) .
\end{aligned}
$$

In all of the above equations we have used the sign function $\xi$ defined as

$$
\xi\left(\ell_{p}+\ell_{j}+J\right) \equiv \frac{1+(-1)^{\ell_{p}+\ell_{h}+J}}{2} .
$$

The functions, $R_{p}\left(R_{h}\right)$ represent the radial single-particle (single-hole) wave functions, and we have introduced the term $\gamma_{i} \equiv\left(\ell_{i}-j_{i}\right)\left(2 j_{i}+1\right)$. 


\section{Appendix C: Fermi Gas Model}

In this appendix we summarize the main features of the relativistic Fermi gas model (RFG), drawing on the detailed discussions in Refs. [9, 10, 11, 12, 13]. The purpose in presenting this material again is twofold: first, we make comparisons with the RFG and its extensions [13] when discussing PV electron scattering in the results section of the present paper; secondly, there are approximations that we make when employing the CSM and the RFG approach provides the basic motivation for these, as discussed below.

We begin by re-stating the procedures followed in deriving the RFG responses in the abovecited work. The exact, fully-relativistic, on-shell, single-nucleon current matrix elements obtained by inserting spin spinors before and after the current operators given in Eqs. (65 70) and (73 75) may be computed in a straightforward way. The results are given in Ref. [11] (in fact, multiplied by factors that occur in the invariant phase-space factors). These are the matrix elements for elastic electroweak scattering from on-shell, moving nucleons. Taking, as usual, the RFG step-function momentum distribution characterized by dimensionless Fermi momentum $\eta_{F} \equiv p_{F} / M$ and integrating over all nucleons in the filled Fermi sea, one obtains the familiar response functions

$$
\begin{aligned}
R^{L, T} & =R_{0}(\kappa, \lambda) U^{L, T}(\kappa, \lambda) \\
R_{A V}^{L, T} & =R_{0}(\kappa, \lambda) \tilde{U}^{L, T}(\kappa, \lambda) \\
R_{V A}^{T^{\prime}} & =R_{0}(\kappa, \lambda) \tilde{U}^{T^{\prime}}(\kappa, \lambda)
\end{aligned}
$$

where the dimensionless momentum and energy transfers are defined in Appendix A. One may consider both the Pauli-blocked and non-Pauli-blocked regimes $\left(\kappa<\eta_{F}\right.$ and $\kappa>\eta_{F}$, respectively). Focusing here on the latter and following Ref. [10] we define a scaling variable

$$
\begin{aligned}
\psi \equiv\left[\frac{1}{\xi_{F}}\left(\kappa \sqrt{1+\frac{1}{\tau}}-(1+\lambda)\right)\right]^{1 / 2} \\
\\
\quad \times\left(\theta\left[\lambda-\lambda_{0}\right]-\theta\left[\lambda_{0}-\lambda\right]\right),
\end{aligned}
$$

where $\xi_{F} \equiv \sqrt{1+\eta_{F}^{2}}-1 \cong \eta_{F}^{2} / 2$, since $\eta_{F}$ is typically small, as discussed in Appendix A. The RFG QE peak occurs at $\lambda=\lambda_{0} \equiv \frac{1}{2}\left[\sqrt{1+4 \kappa^{2}}-1\right]$.

In the non-Pauli-blocked regime the overall responses are characterized by

$$
R_{0}(\kappa, \lambda)=\frac{3 \mathcal{N} \xi_{F}}{4 M \kappa \eta_{F}^{3}}\left(1-\psi^{2}\right) \theta\left(1-\psi^{2}\right),
$$

where $\mathcal{N}$ is $Z(N)$ when protons (neutrons) are involved (the expressions for the Pauli-blocked regime are discussed in Ref. [10] - here our main emphasis is on the non-Pauli-blocked region although in Sect. 3 some results are also presented for $\kappa<\eta_{F}$ ). The dependences on the 
single-nucleon content in the problem are then isolated in the factors

$$
\begin{aligned}
U^{L}(\kappa, \lambda) & =\frac{\kappa^{2}}{\tau}\left\{\left[(1+\tau) W_{2}(\tau)-W_{1}(\tau)\right]+W_{2}(\tau) \Delta\right\} \\
U^{T}(\kappa, \lambda) & =\frac{\kappa^{2}}{\tau}\left\{\left[(1+\tau) \tilde{W}_{2}(\tau)-\tilde{W}_{1}(\tau)\right]+\tilde{W}_{2}(\tau) \Delta\right\} \\
\tilde{U}^{L}(\kappa, \lambda) & =2 W_{1}(\tau)+W_{2}(\tau) \Delta \\
\tilde{U}^{T}(\kappa, \lambda) & =2 \tilde{W}_{1}(\tau)+\tilde{W}_{2}(\tau) \Delta \\
\tilde{U}^{T^{\prime}}(\kappa, \lambda) & =\sqrt{\tau(1+\tau)} \tilde{W}_{3}(\tau)\{1+\tilde{\Delta}\} .
\end{aligned}
$$

Here the nucleon form factors are those discussed in the main body of the text (for protons or neutrons, as is appropriate). The factors $\Delta$ and $\tilde{\Delta}$ were introduced in Refs. [9, 10]:

$$
\begin{aligned}
\Delta & =\frac{\tau}{\kappa^{2}} \xi_{F}\left(1-\psi^{2}\right)\left\{\kappa \sqrt{1+\frac{1}{\tau}}+\frac{1}{3} \xi_{F}\left(1-\psi^{2}\right)\right\} \\
\tilde{\Delta} & =\frac{1}{\kappa} \sqrt{\frac{\tau}{1+\tau}}\left\{\frac{1}{2} \xi_{F}\left(1-\psi^{2}\right)\right\} .
\end{aligned}
$$

Clearly these two terms provide relatively small contributions to the totals in Eqs. (98 102), since they both involve the factor $\xi_{F}$ which is very small. These are contributions of order $\eta_{F}^{2}$ and typically enter at the few percent level in the kinematic regime of interest here. Indeed, in Appendix A we argued that such $\mathcal{O}\left(\eta^{2}\right)$ effects could be neglected - we have done so in the treatment of the CSM in the present work. In Sect. 3.1 we present results for the RFG (and these agree with the past results cited above) both with the exact relativistic current matrix elements and with the approximations discussed in Appendix A. Moreover, we also display in Sect. 3.1 some results for what we defined in Appendix A to be the strict non-relativistic approximation (see Eqs. (82 84)) to illustrate how poor those commonly-made approximations can be (see Fig. 2).

A second point to be drawn from this summary of the RFG formalism, in addition to bringing out the roles played by the single-nucleon current matrix elements, is seen by returning to $R_{0}$ defined in Eq. (97). The basic RFG behavior in the non-Pauli-blocked region when the singlenucleon factors (the $U^{\prime}$ 's and $\tilde{U}$ 's) are removed is parabolic in the scaling variable $\psi$. For the RFG this quantity ranges between -1 and +1 , with the QE peak occurring when $\psi=0$. An excellent approximation to the scaling variable may be shown to be (see Ref. [12])

$$
\psi \cong \frac{1}{\eta_{F}}\left[\frac{\lambda(1+\lambda)}{\kappa}-\kappa\right],
$$

making it easy to see that the QE peak $(\psi=0)$ corresponds to $\lambda(1+\lambda)=\kappa^{2} \leftrightarrow \lambda=\tau \leftrightarrow \omega=$ $\left|Q^{2}\right| / 2 M$, the relativistic kinematical relation. The non-relativistic version of Eq. (105) is

$$
\psi_{n r} \cong \frac{1}{\eta_{F}}\left[\frac{\lambda}{\kappa}-\kappa\right]
$$


and yields the non-relativistic relation $\lambda=\kappa^{2} \leftrightarrow \omega=q^{2} / 2 M$ for the peak position. In the past Fermi gas work cited above it was shown that a very good approximation is to employ the non-relativistic many-body responses (of course, using good single-nucleon currents), but with the replacement $\lambda \rightarrow \lambda(1+\lambda)$ or, for dimensionful variables, $\omega \rightarrow \omega(1+\omega / 2 M)$. The replacement is made only in the many-body aspects of the problem, not in the single-nucleon form factors which are evaluated at the correct value of $\tau$, namely that given by the electron kinematic variables. In Sect. 3.1 we present results to illustrate the quality of this replacement in the nuclear kinematics. With this as motivation, we follow the same "relativizing" procedure for the kinematics of the CSM as well by using the relativistic relation in the energy-conserving $\delta$-function by making the same replacement.

\section{References}

[1] E. G. Adelberger and W. C. Haxton, Ann. Rev. Nucl. Part. Sci. 35 (1985) 501

[2] See for instance B. Desplanques, J. F. Donoghue and B. R. Holstein, Ann. Phys. 124 (1980) 449

[3] W. C. Haxton, E. M. Henley and M. J. Musolf, Phys. Rev. Lett. 63 (1989) 949

[4] F. J. Hasert et al., Phys. Lett. 46B (1973) 138

[5] C. Y. Prescott et al., Phys. Lett. 77B (1978) 347

[6] M. J. Musolf et al., Phys. Rep. 239 (1994) 1

[7] P. A. Souder et al., Phys. Rev. Lett. 65 (1990) 694

[8] W. Heil et al., Nucl. Phys. B327 (1989) 1

[9] T. W. Donnelly et al., Nucl. Phys. A541 (1992) 525

[10] W. M. Alberico et al., Phys. Rev. C38 (1988) 1801

[11] W. M. Alberico, T. W. Donnelly and A. Molinari, Nucl. Phys. A512 (1990) 541

[12] W. M. Alberico et al., Nucl. Phys. A563 (1993) 605

[13] M. B. Barbaro et al., Nucl. Phys. A569 (1994) 701

[14] C. J. Horowitz, Phys. Rev. C47 (1993) 826; C. J. Horowitz and J. Piekarewicz, Phys. Rev. C47 (1993) 2924 
[15] J. Martorell and E. Moya de Guerra, Ann. Phys. 158 (1984) 1

[16] J. E. Amaro, G. Co' and A. M. Lallena, Ann. Phys. 221 (1993) 306; J. E. Amaro, G. Co', E. Fasanelli and A. M. Lallena, Phys. Lett. B277 (1992) 249

[17] J. E. Amaro, G. Co' and A. M. Lallena, Nucl. Phys. A578 (1994) 365.

[18] J. E. Amaro, A. M. Lallena and G. Co', Int. J. Mod. Phys. E3 (1994) 735

[19] G. Co', K. Q. Quader, R. Smith and J. Wambach, Nucl. Phys. A485 (1988) 61

[20] D. Pines, K. Q. Quader and J. Wambach, Nucl. Phys. A469 (1988) 365

[21] M. J. Musolf and T. W. Donnelly, Nucl. Phys. A546 (1992) 509

[22] J. D. Bjorken and S. D. Drell, Relativistic Quantum Mechanics, McGraw-Hill (1964)

[23] S. Galster et al., Nucl. Phys. B32 (1971) 221

[24] J. E. Amaro, C. García-Recio and A. M. Lallena, Nucl. Phys. A567 (1994) 701

[25] G. A. Rinker and J. Speth, Nucl. Phys. A306 (1978) 360 and J. Speth, V. Klemt, J. Wambach and G. E. Brown, Nucl. Phys. A343 (1980) 382

[26] J. E. Amaro, Ph.D. Thesis, University of Granada (1993) unpublished

[27] T. de Forest, Jr. and J. D. Walecka, Adv. in Phys. 15 (1966) 1

[28] E. Hadjimichael, G. I. Poulis and T. W. Donnelly, Phys. Rev. C45 (1992) 2666

[29] M. B. Barbaro et al., (to be published) 


\begin{tabular}{rrrrrrrr} 
& & $V_{0}[\mathrm{MeV}]$ & $V_{l s}[\mathrm{MeV}]$ & $R_{0}[\mathrm{fm}]$ & $a_{0}[\mathrm{fm}]$ & $R_{l s}[\mathrm{fm}]$ & $a_{l s}[\mathrm{fm}]$ \\
\hline${ }^{16} \mathrm{O}$ & $\mathrm{p}$ & 52.5 & 7.00 & 3.20 & 0.53 & 3.20 & 0.53 \\
& $\mathrm{n}$ & 52.5 & 6.54 & 3.20 & 0.53 & 3.20 & 0.53 \\
${ }^{40} \mathrm{Ca}$ & $\mathrm{p}$ & 57.5 & 11.11 & 4.10 & 0.53 & 4.10 & 0.53 \\
& $\mathrm{n}$ & 55.0 & 8.50 & 4.10 & 0.53 & 4.10 & 0.53 \\
${ }^{208} \mathrm{~Pb}$ & $\mathrm{p}$ & 60.4 & 6.75 & 7.46 & 0.79 & 7.20 & 0.59 \\
& $\mathrm{n}$ & 44.3 & 6.08 & 7.46 & 0.66 & 6.96 & 0.64 \\
\hline
\end{tabular}

Table 1: Parameters of the Woods-Saxon potential. They have been taken from Refs. 18 for ${ }^{16} \mathrm{O}$, 24] for ${ }^{40} \mathrm{Ca}$, and [25] for ${ }^{208} \mathrm{~Pb}$.

\begin{tabular}{cccc} 
Nucleus & $q[\mathrm{MeV} / \mathrm{c}]$ & $J_{\text {max }}$ & $J_{\text {con }}$ \\
\hline \hline${ }^{16} \mathrm{O}$ & 300 & 15 & 8 \\
& 500 & 15 & 12 \\
& 700 & 20 & 20 \\
\hline${ }^{40} \mathrm{Ca}$ & 300 & 15 & 11 \\
& 500 & 15 & 15 \\
& 700 & 23 & 23 \\
\hline${ }^{208} \mathrm{~Pb}$ & 300 & 20 & 18 \\
& 500 & 23 & 23 \\
& 700 & 30 & 30 \\
\hline
\end{tabular}

Table 2: Multipoles used in the calculation. 


\section{FIGURE CAPTIONS}

1. One boson exchange diagrams considered in this work.

2. Electromagnetic response functions $R^{L}$ and $R^{T}$ for ${ }^{40} \mathrm{Ca}$ shown at $q=500,700$ and 1000 $\mathrm{MeV} / \mathrm{c}$ : (solid curves) full RFG model; using the approximate currents given in Sect. 2.2, (long-dashed curves) with the replacement $\lambda \rightarrow \lambda(1+\lambda)$ made to the non-relativistic FG model as discussed in Appendix $\mathrm{C}$ or (dot-dashed curves) without this replacement; as in the previous case, but using the non-relativistic currents obtained by setting the factor $\kappa / \sqrt{\tau} \rightarrow 1$ in Eqs. (20) and (22) with (short-dashed curves) or without (dotted curves) the $\lambda \rightarrow \lambda(1+\lambda)$ replacement. In all cases $p_{F}$ is fixed at $235 \mathrm{MeV} / \mathrm{c}$.

3. Electromagnetic response functions $R^{L}$ and $R^{T}$ for ${ }^{16} \mathrm{O}$ at $q=300,500$ and $700 \mathrm{MeV} / \mathrm{c}$ : (solid curves) CSM; (other curves) full RFG model, (dashed curves, $p_{F}=215 \mathrm{MeV} / \mathrm{c}$ ) and (dotted curves, $p_{F}=225 \mathrm{MeV} / \mathrm{c}$ ).

4. Electromagnetic response function $R^{L}$ for ${ }^{40} \mathrm{Ca}$ and ${ }^{208} \mathrm{~Pb}$ at $q=300,500$ and $700 \mathrm{MeV} / \mathrm{c}$. The labeling in this and most of the following figures is: (solid curves) CSM; (other curves) full RFG model. The latter are the following: for ${ }^{40} \mathrm{Ca}$ two are shown, (dashed curves, $p_{F}=235 \mathrm{MeV} / \mathrm{c}$ ) and (dotted curves, $245 \mathrm{MeV} / \mathrm{c}$ ); for ${ }^{208} \mathrm{~Pb}$ three are shown, (long-dashed curves, $p_{F p}=250 \mathrm{MeV} / \mathrm{c}, p_{F n}=288 \mathrm{MeV} / \mathrm{c}$ ), (short-dashed curves, $p_{F p}=p_{F n}=250$ $\mathrm{MeV} / \mathrm{c}$ ) and (dotted curves, $p_{F p}=230 \mathrm{MeV} / \mathrm{c}, p_{F n}=265 \mathrm{MeV} / \mathrm{c}$ ), as discussed in the text. In fact, for the longitudinal response the short- and long-dashed curves essentially coincide.

5. As in Fig. 4, except now for the EM response $R^{T}$.

6. As in Fig. 4, except now for the PV response $R_{A V}^{T}$.

7. As in Fig. 4, except now for the PV response $R_{V A}^{T^{\prime}}$.

8. As in Fig. 4, except now for the PV response $R_{A V}^{L}$.

9. Parity-violating asymmetry for ${ }^{16} \mathrm{O},{ }^{40} \mathrm{Ca}$ and ${ }^{208} \mathrm{~Pb}$ at $q=300,500$ and $700 \mathrm{MeV} / \mathrm{c}$ and at $\theta_{e}=10^{\circ}$ and $150^{\circ}$ (labeling of curves as in Fig. 4). The difficulty in distinguishing the curves shows the nuclear model insensitivity of the results.

10. The sum-rule asymmetry (SRA) defined in Eq. (57) shown for ${ }^{40} \mathrm{Ca}$ and ${ }^{208} \mathrm{~Pb}$ at $q=300$, 500 and $700 \mathrm{MeV} / \mathrm{c}$ as a function of scattering angle $\theta_{e}$ (labeling of curves as in Fig. 4). The difficulty in distinguishing the curves, especially in the case of ${ }^{40} \mathrm{Ca}$, reflects the high degree of nuclear model insensitivity for this observable. 
11. The sum-rule asymmetry (SRA) defined in Eq. (57) shown for ${ }^{16} \mathrm{O}$ (solid curves), ${ }^{40} \mathrm{Ca}$ (dashed curves) and ${ }^{208} \mathrm{~Pb}$ (dotted curves) at $q=300,500$ and $700 \mathrm{MeV} / \mathrm{c}$ as a function of scattering angle $\theta_{e}$. The CSM is used in all three cases. The difficulty in distinguishing the curves (the results for ${ }^{16} \mathrm{O}$ and ${ }^{40} \mathrm{Ca}$ are essentially equal) indicates the universality of this observable for a wide range of nuclei.

12. As in Fig. 10, but now showing families of curves with magnetic strangeness (with $\mu_{s}=$ -0.35 ) and without magnetic strangeness (indicated with a "0" in the case of ${ }^{40} \mathrm{Ca}$ where the families of curves can be distinguished at high $q$ ). In this case the curves are seen to coalesce into sets determined by the value of $\mu_{s}$ and not by the nuclear model variations, as discussed in more depth in the text. The families of curves are labeled as in Fig. 4.

13. As in Fig. 10, but now focusing on scattering at backward angles and showing families of curves with different values of the isovector, axial-vector form factor of the nucleon. The curves marked with a $( \pm)$ correspond to setting $g_{A}=1.262 \pm 10 \%$, while the remaining curves have this quantity set to 1.262. The families of curves are labeled as in Fig. 4. At intermediate-to-high values of $q$ the curves are seen to group into sets determined by the axial-vector coupling and not so much by the nuclear model variations.

14. As in Fig. 10, but now focusing on scattering at forward angles and showing families of curves with different values of the neutron electric form factor, the standard Galster parameterization with $\lambda_{n}=5.6$ (see Ref. [6]) and values of this parameter that yield \pm $10 \%$ variation in $G_{E}^{n}$ at kinematics where the form factor peaks, namely $\lambda_{n}=4.2$ and 7.3 (marked $( \pm)$ in the figure when the groups are distinguishable). The families of curves are labeled as in Fig. 4. The nuclear model dependences and the differences due to variations in $G_{E}^{n}$ at this level would clearly be quite difficult to separate.

15. As in Fig. 14, but now showing families of curves with different parameterizations for $G_{E}^{(s)}$ : the curves marked (I) have no $G_{E}^{(s)}$ contributions, marked (II) have $\rho_{s}=-3$ and $\lambda_{E}^{(s)}=5.6$ ("modest electric strangeness"), while those marked (III) have $\rho_{s}=-3$ and $\lambda_{E}^{(s)}=0$ ("large electric strangeness"). In all cases the magnetic strangeness form factor has been taken to be the standard parameterization discussed in the text. Clearly for high $q$ and forward angles the nuclear model dependences are considerably weaker than the dependence on the parameterization of electric strangeness in the nucleon. 


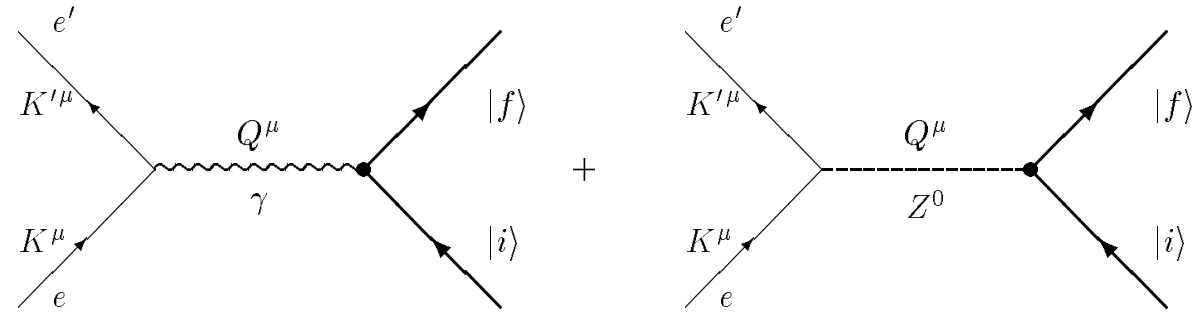


${ }^{40} \mathrm{Ca}$
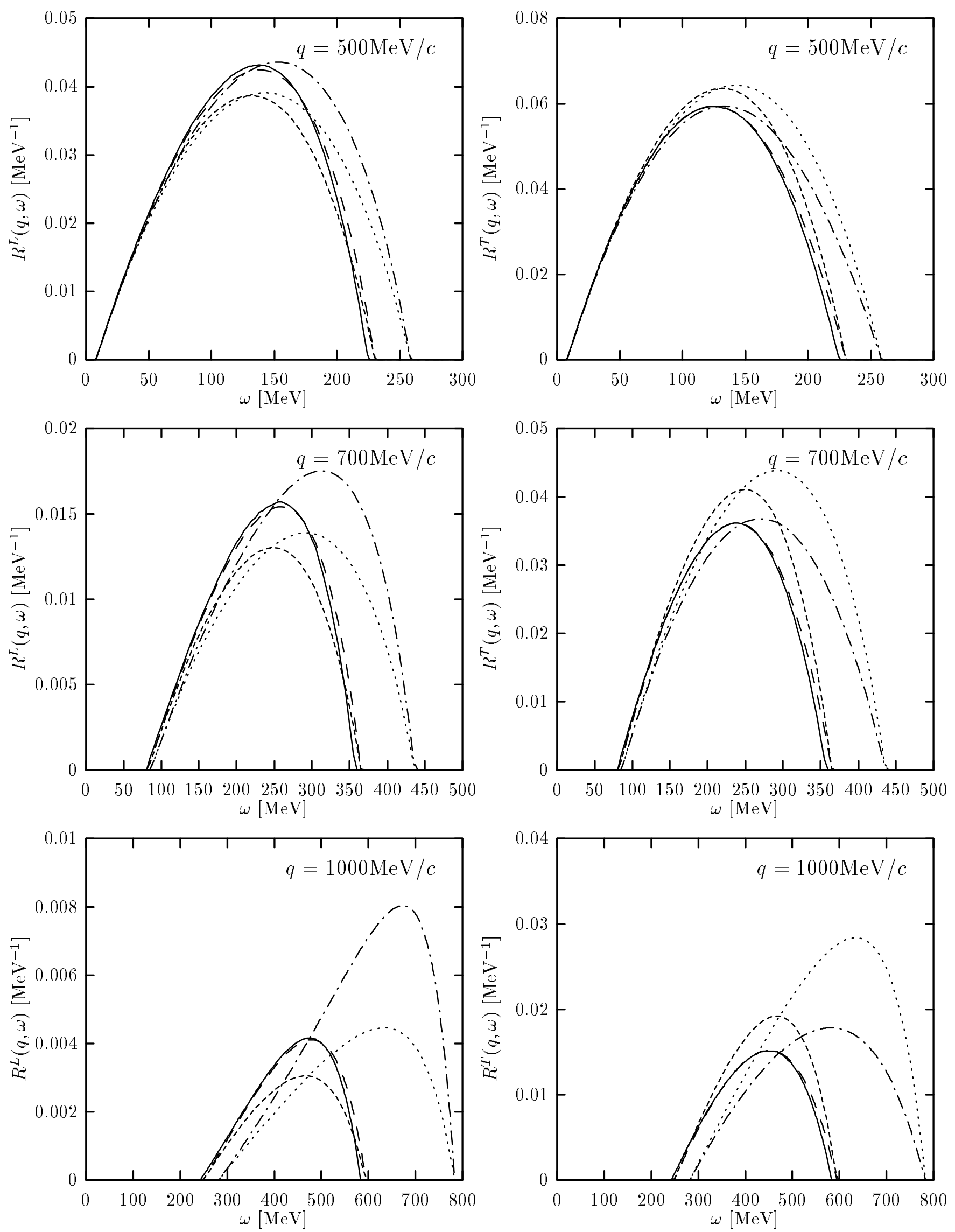

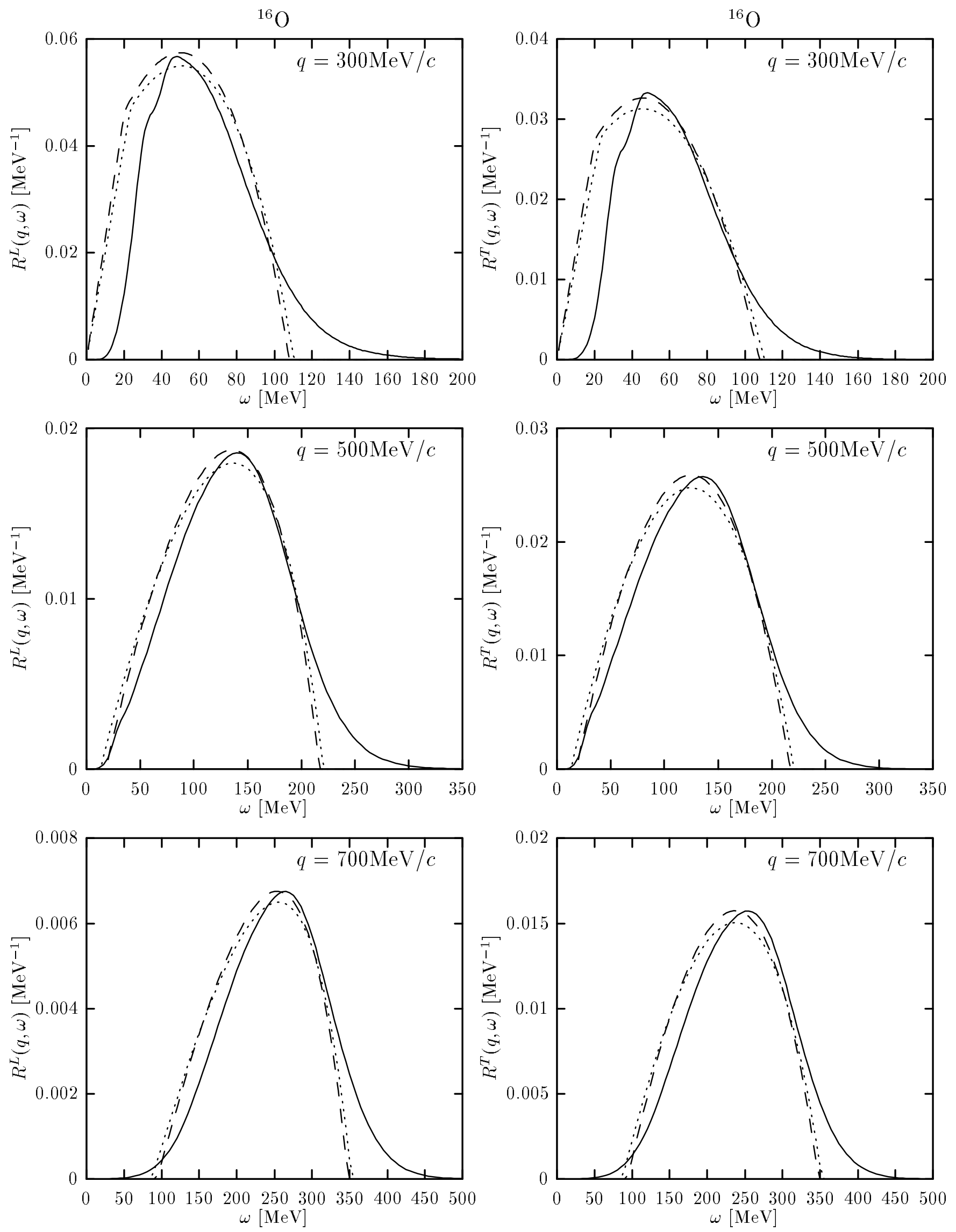

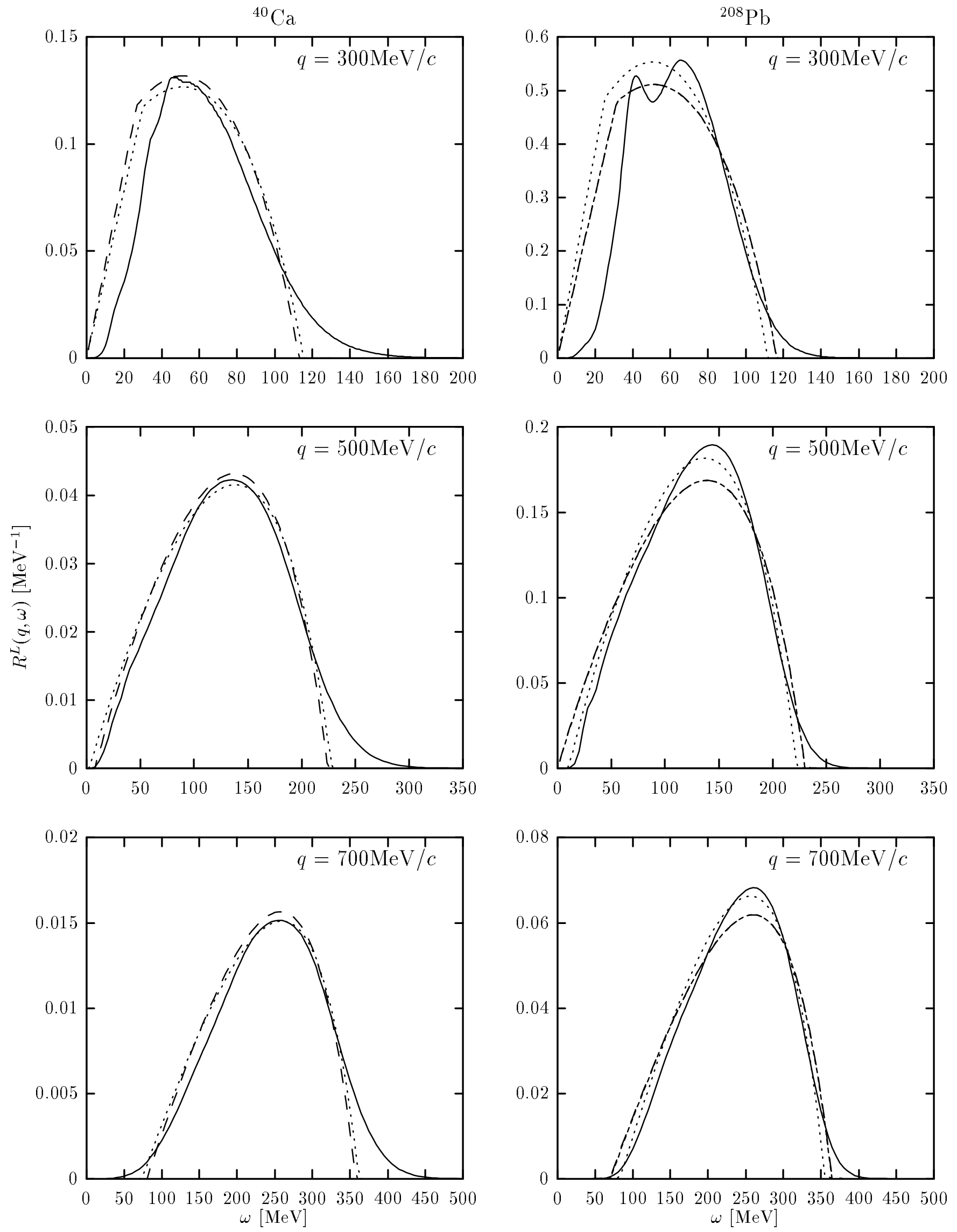

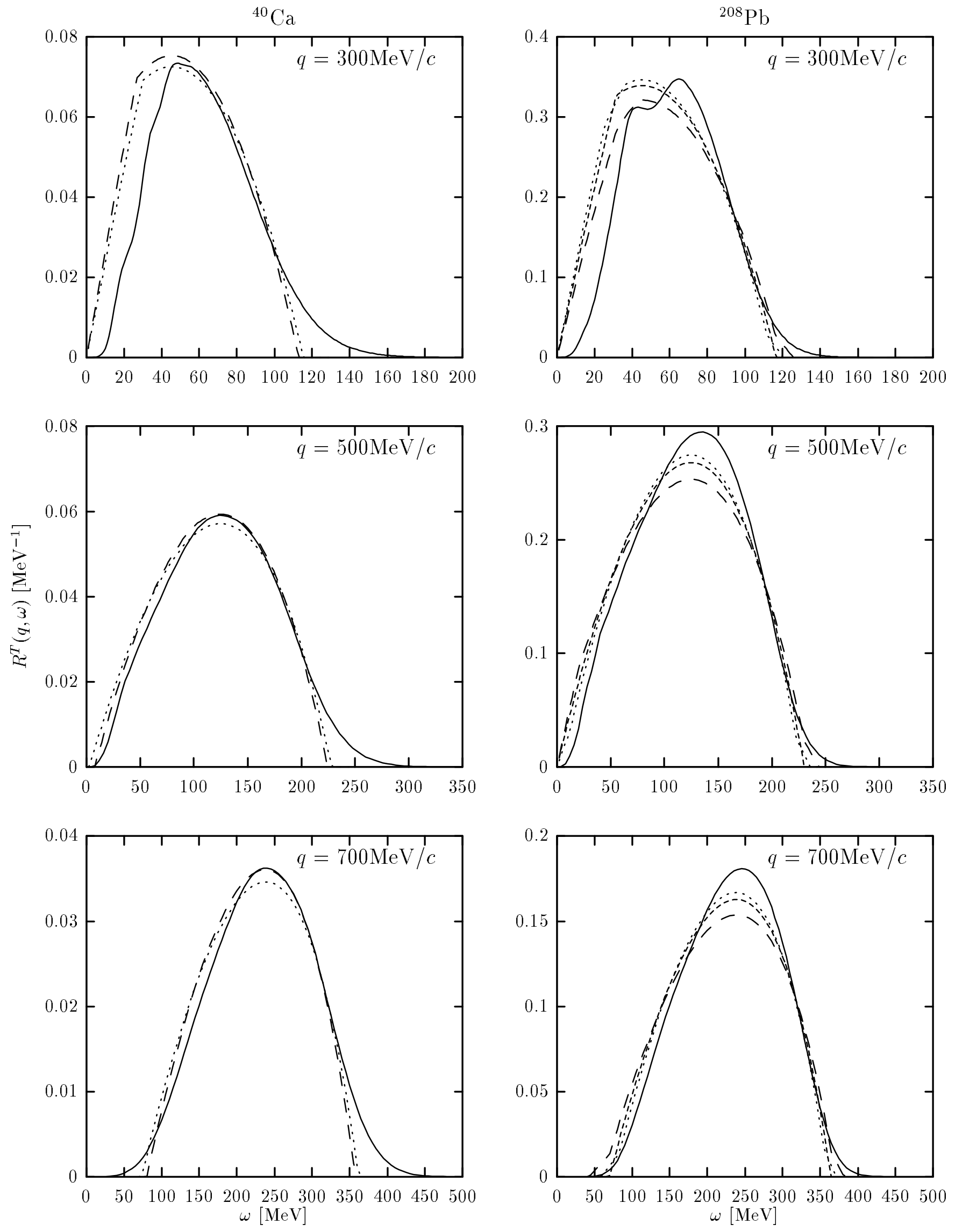

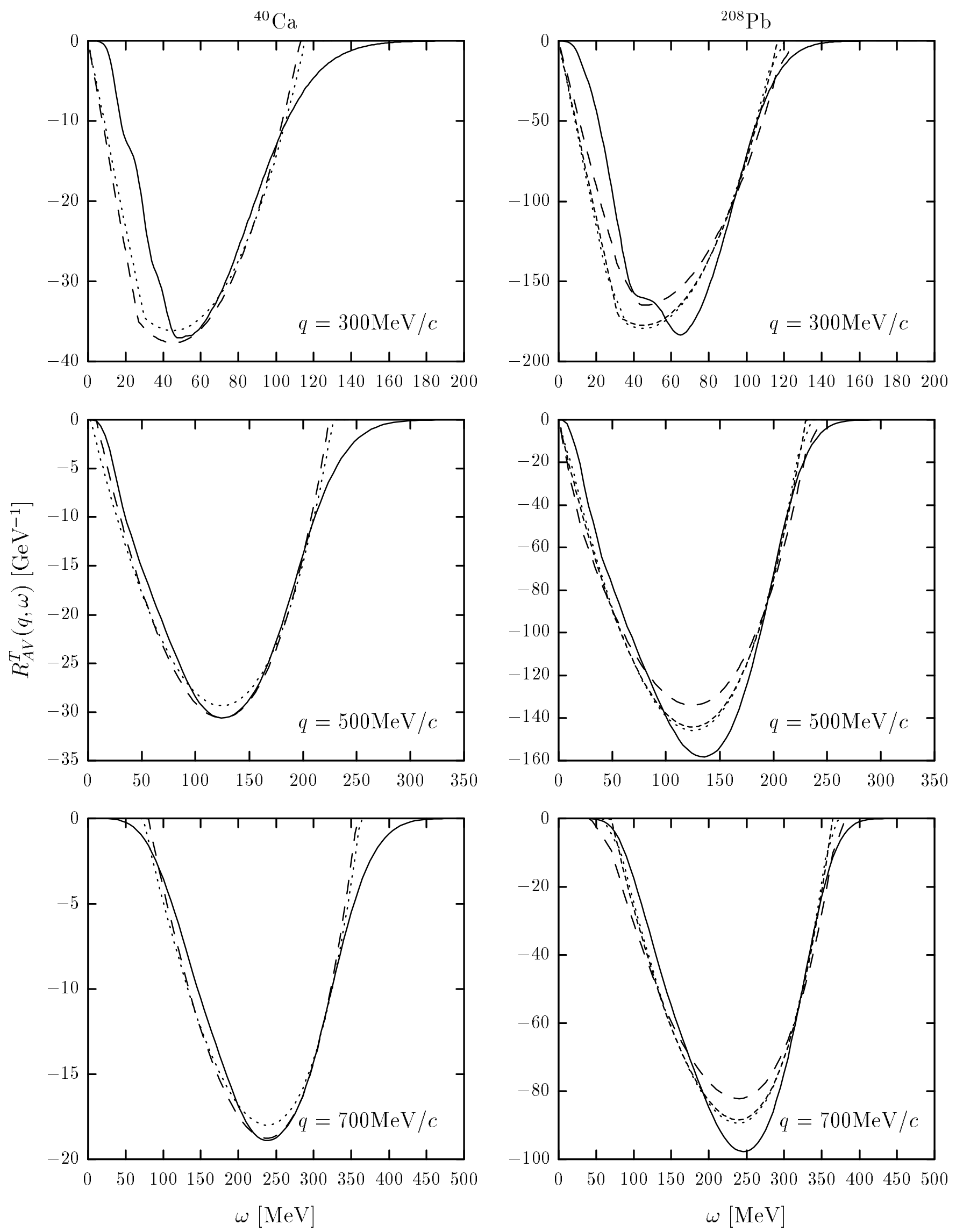

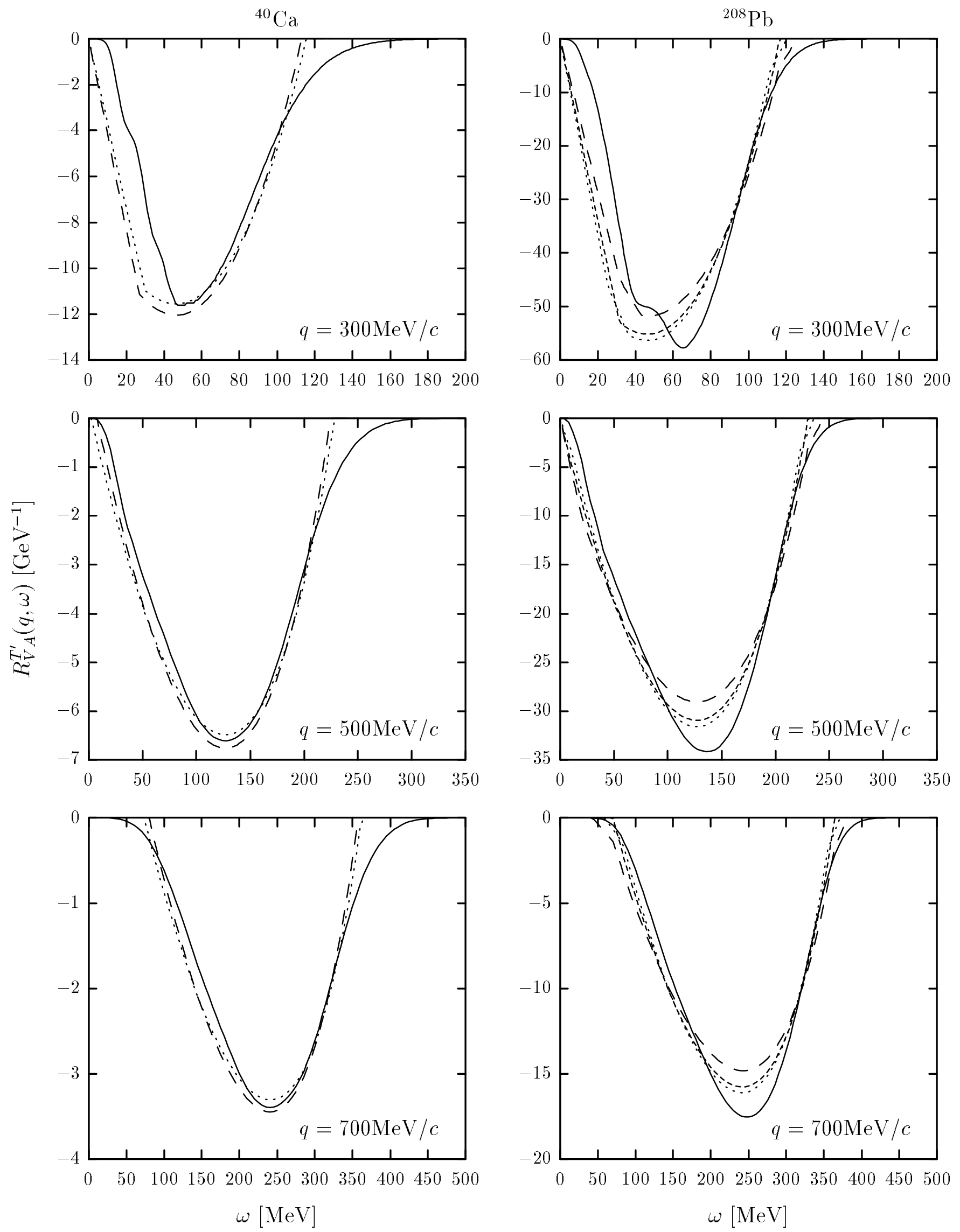

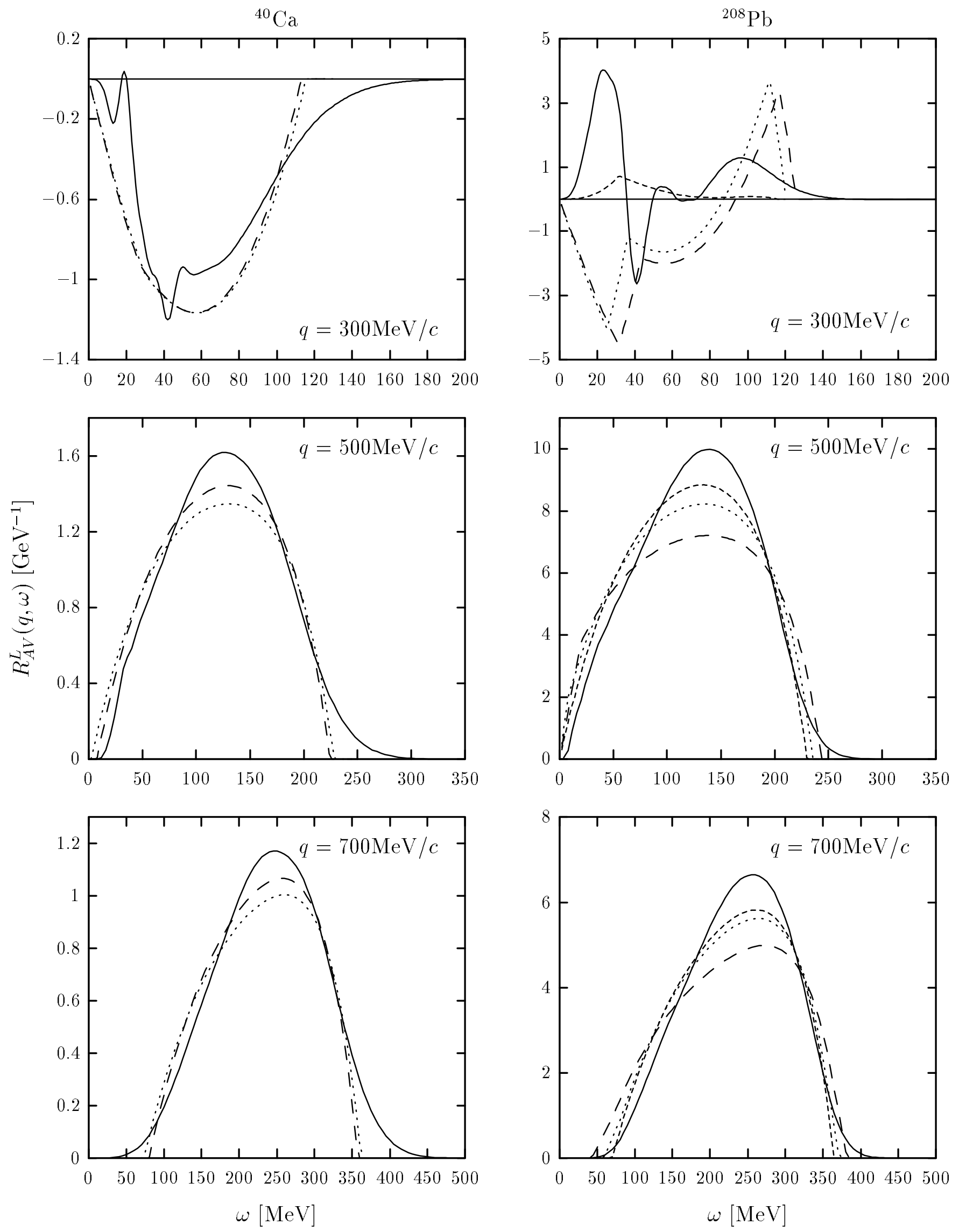

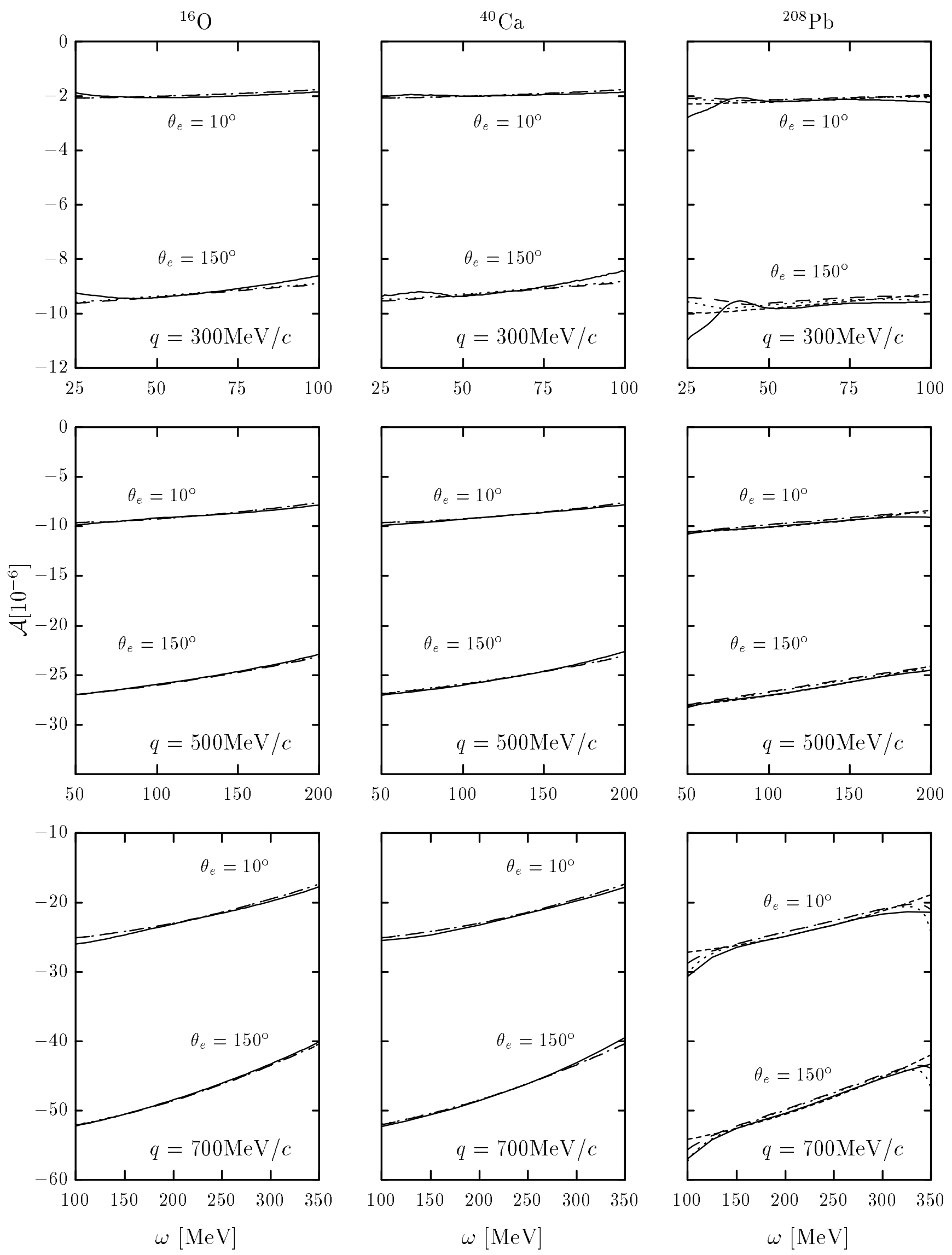

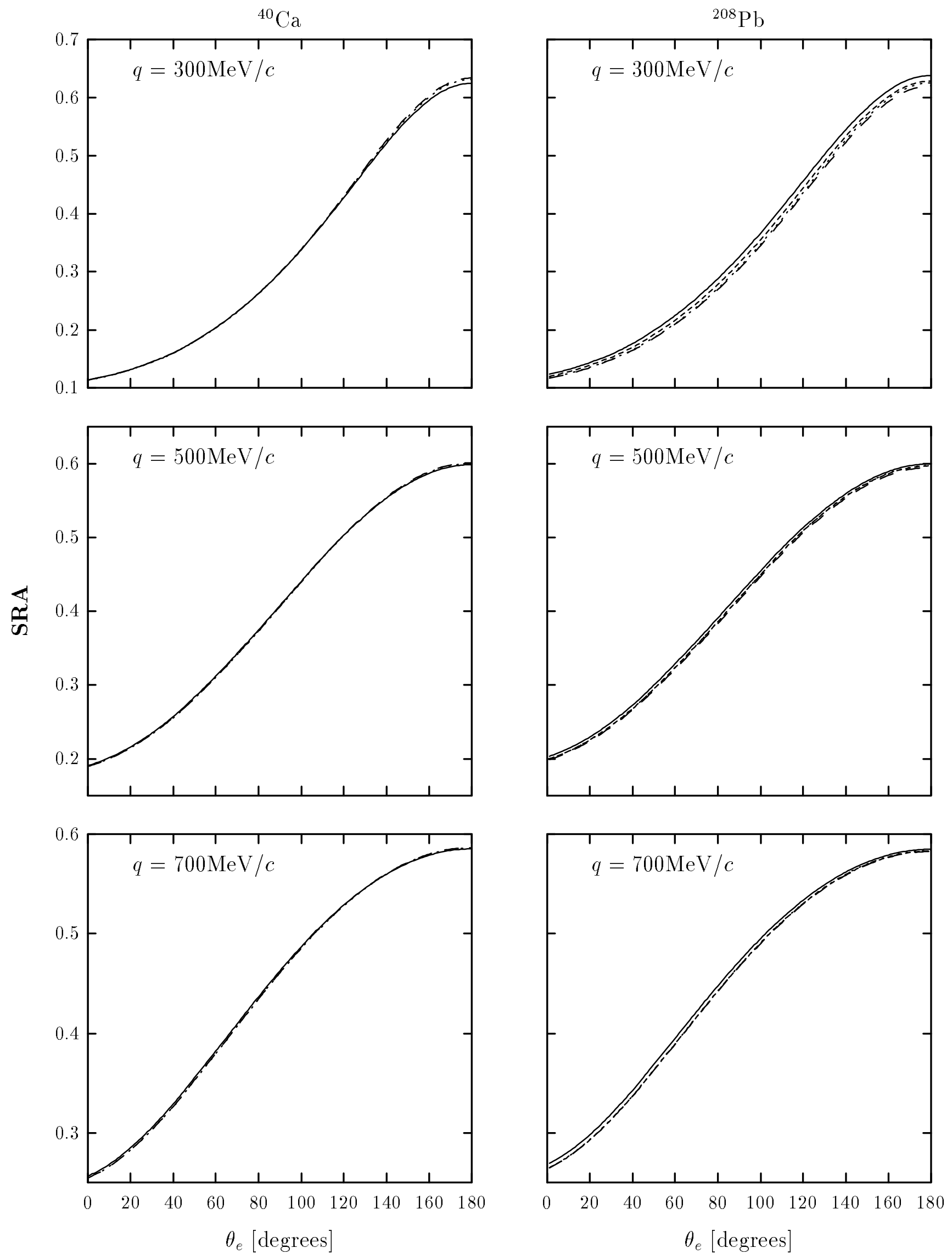


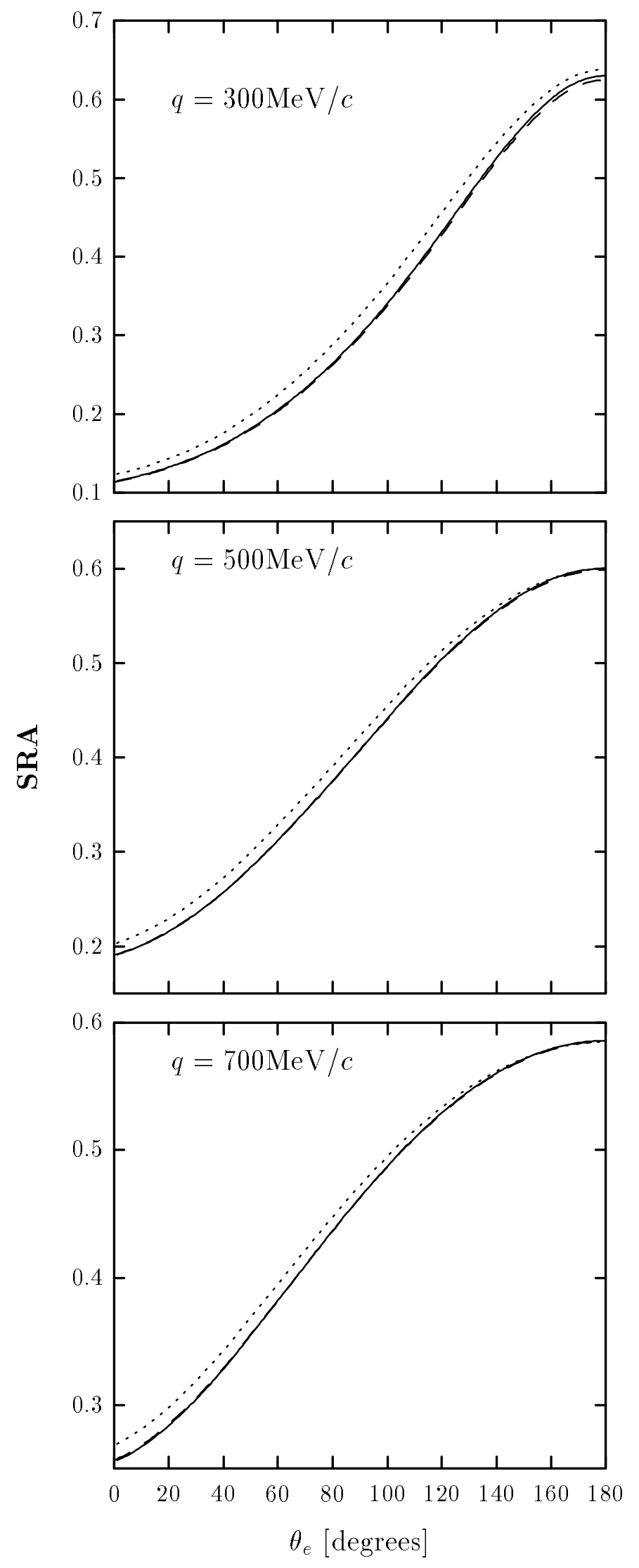



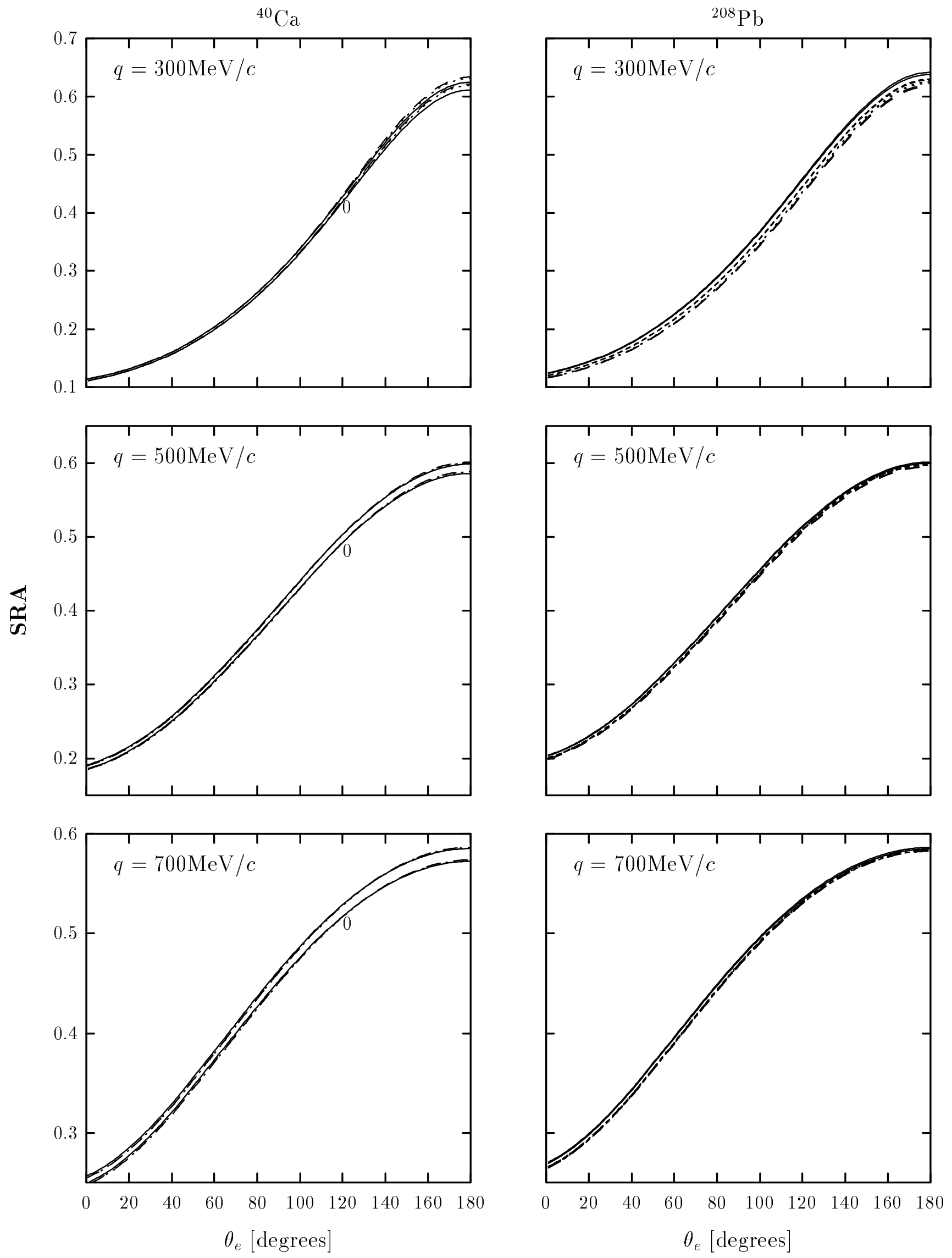

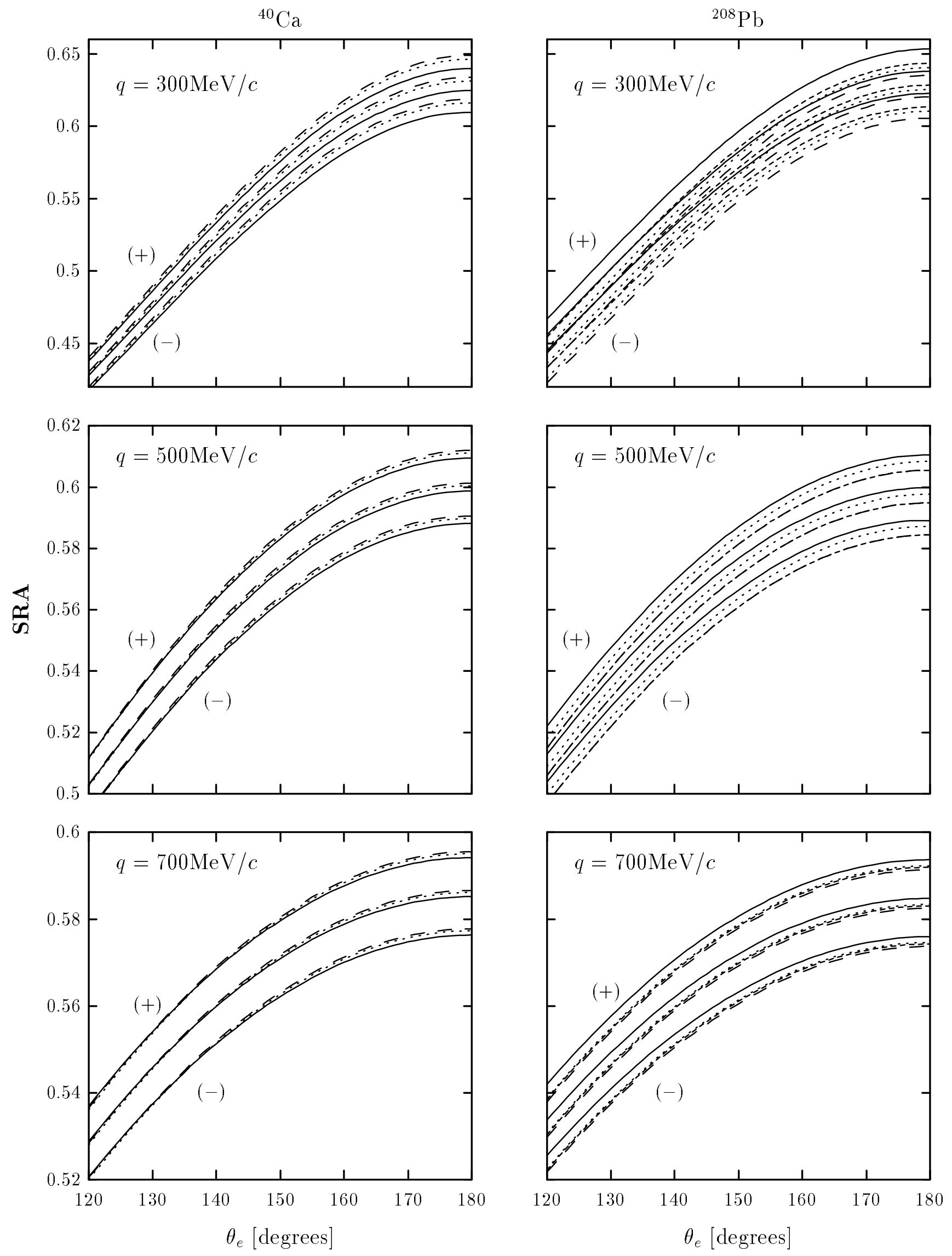

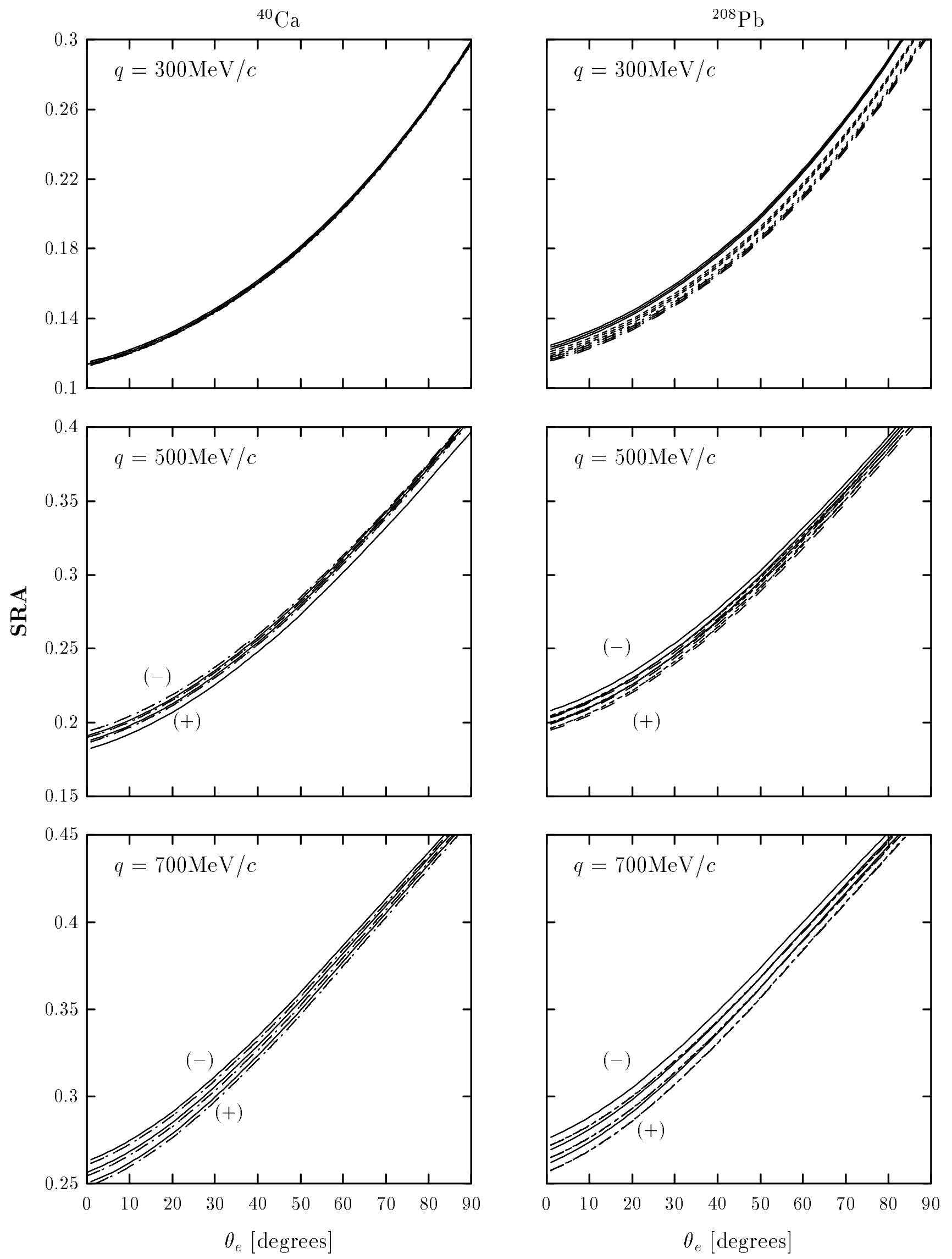

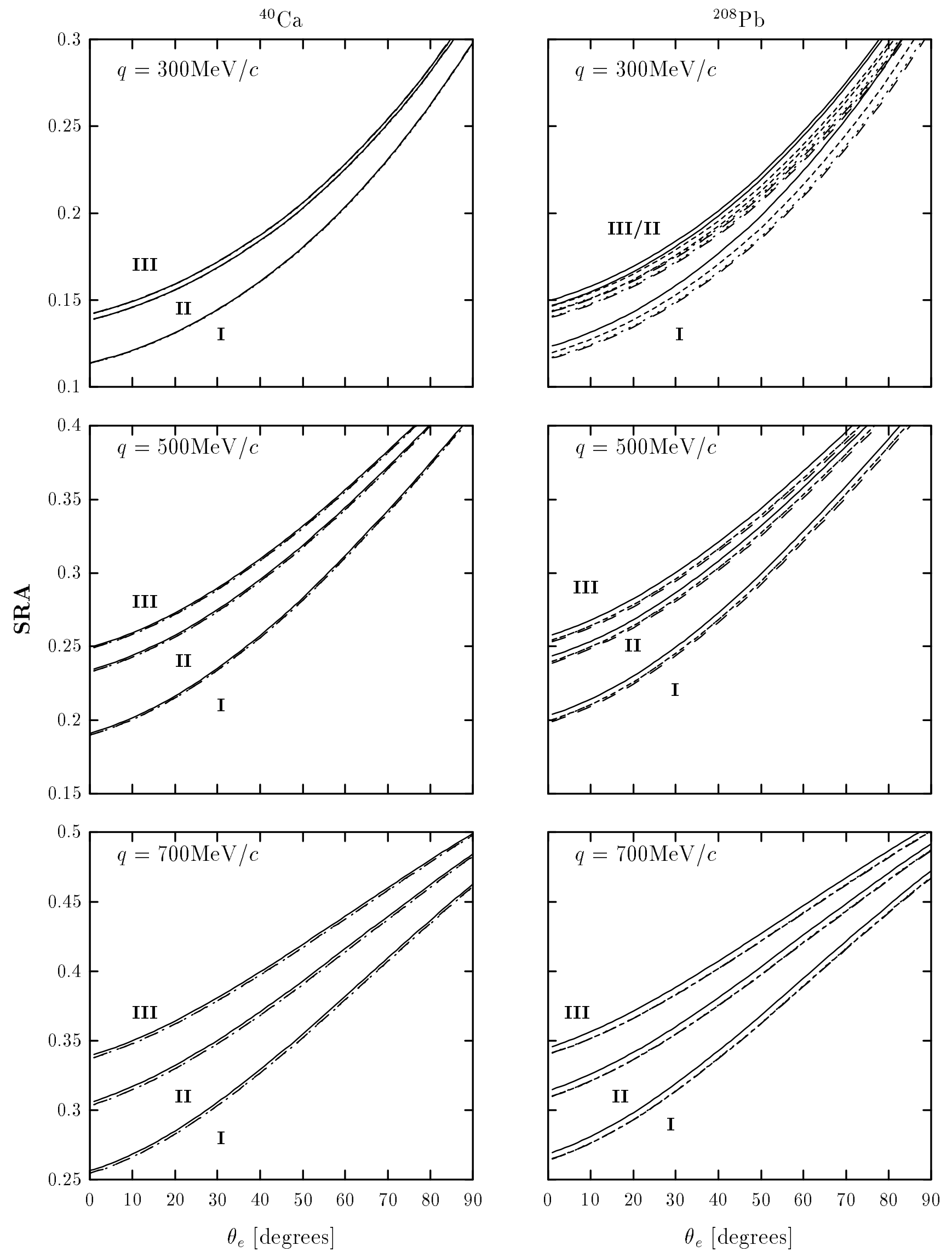